\title{
SQUARE FUNCTIONS, BOUNDED ANALYTIC SEMIGROUPS, AND APPLICATIONS
}

\author{
CHRISTIAN LE MERDY \\ Département de Mathématiques, Université de Franche-Comté \\ 25030 Besançon Cedex, France \\ E-mail: lemerdy@math.univ-fcomte.fr
}

\begin{abstract}
To any bounded analytic semigroup on Hilbert space or on $L^{p}$-space, one may associate natural 'square functions'. In this survey paper, we review old and recent results on these square functions, as well as some extensions to various classes of Banach spaces, including noncommutative $L^{p}$-spaces, Banach lattices, and their subspaces. We give some applications to $H^{\infty}$ functional calculus, similarity problems, multiplier theory, and control theory.
\end{abstract}

1. Introduction. Square functions for generators of semigroups grew out of classical harmonic analysis, and the Littlewood-Paley theory. They were first developed in Stein's classical book [52], with applications to functional calculus and multiplier theorems for diffusion semigroups on $L^{p}$-spaces. Later on, further remarkable extensions of these multiplier theorems were obtained by Cowling in [10], who used them to obtain maximal theorems. Then in the three fundamental papers [42, 43, 11], McIntosh and his coauthors introduced $H^{\infty}$ functional for sectorial operators on Banach space $X$, generalized associated square functions if $X$ is a Hilbert space or an $L^{p}$-space, and found deep connections between these topics, giving a new viewpoint on Stein and Cowling's results. Since then, $H^{\infty}$ calculus and its applications to evolution equations and semigroups have developed rapidly.

The purpose of this survey paper is to give a review of the various connections between functional calculus and square functions, as well as some of their applications to several aspects of the theory of semigroups. Of course it is only a selection of these applications, obviously influenced by the author's tastes.

In Section 2, we briefly review all necessary definitions and basic results concerning sectorial operators, $H^{\infty}$ functional calculus, and bounded analytic semigroups. We also include a subsection on Rademacher boundedness, a recent notion which now plays a key

2000 Mathematics Subject Classification: 47A60, 47D03.

The paper is in final form and no version of it will be published elsewhere. 
role in the development of the subject (see e.g. [29, 30, 35, 56, 57]). Section 3 is devoted to square functions on Hilbert space. It is mainly based on [42, 43]. We give proofs of the main results so that the reader can gain some intuition on the subject. Indeed, from these results grew many generalizations from Hilbert space to broader classes of Banach spaces, which are considered later on in the paper. In Section 4, we give two specific applications of square functions for bounded analytic semigroups on Hilbert space. These applications are independent of each other and show the power of the subject. Subsection 4.1 is devoted to the topic of similarity to contractions, whereas Subsection 4.2 is devoted to the study of operators which are admissible for the generator of a bounded semigroup, a problem arising from control theory. Square functions on $L^{p}$-spaces are introduced in Section 5. We explain Stein and Cowling's original results for diffusion semigroups in this context. The last two sections are devoted to more recent developments of square functions. In Section 6, we review some work from [26, 27] on square functions and semigroups on noncommutative $L^{p}$-spaces associated with a semifinite von Neumann algebra. This includes applications to noncommutative diffusion semigroups and noncommutative Fourier multipliers on free groups. Section 7 contains further extensions of square functions, either in a concrete or in an abstract form.

\section{Preliminaries and the $H^{\infty}$ functional calculus}

2.A. Sectorial operators and their $H^{\infty}$ functional calculus. In the first three subsections, we will define sectorial operators and $H^{\infty}$ functional calculus, and review some of their basic properties. The definitions and results below essentially go back to [42] and [11]. See also [1] or [33] for further details.

Let $X$ be a complex Banach space. We let $B(X)$ denote the Banach algebra of all bounded operators on $X$. The identity operator on $X$ will be either denoted by $I_{X}$ or 1. Let $A$ be a closed and densely defined operator on $X$. We let $D(A), N(A)$, and $R(A)$ denote the domain, the kernel and the range of $A$ respectively. Further we let $\sigma(A)$ and $\rho(A)$ denote the spectrum and the resolvent set of $A$ respectively. For any $\lambda \in \rho(A)$, we let $R(\lambda, A)=(\lambda-A)^{-1}$ be the associated resolvent operator; this is an element of $B(X)$. For any $\omega \in(0, \pi)$, we introduce

$$
\Sigma_{\omega}=\left\{z \in \mathbb{C}^{*}:|\operatorname{Arg}(z)|<\omega\right\},
$$

the open sector of angle $2 \omega$ around the half-line $(0, \infty)$. By definition, $A$ is sectorial of type $\omega$ if $\sigma(A) \subset \overline{\Sigma_{\omega}}$ and if for any $\theta \in(\omega, \pi)$ there is a constant $K_{\theta}>0$ such that

$$
\|\lambda R(\lambda, A)\| \leq K_{\theta}, \quad \lambda \in \mathbb{C} \backslash \overline{\Sigma_{\theta}} .
$$

For any $\theta \in(0, \pi)$, we let $H^{\infty}\left(\Sigma_{\theta}\right)$ be the space of bounded analytic functions $f: \Sigma_{\theta} \rightarrow$ $\mathbb{C}$. This is a Banach algebra for the supremum norm

$$
\|f\|_{\infty, \theta}=\sup \left\{|f(z)|: z \in \Sigma_{\theta}\right\} .
$$

Then we let $H_{0}^{\infty}\left(\Sigma_{\theta}\right)$ be the subalgebra of all $f \in H^{\infty}\left(\Sigma_{\theta}\right)$ for which there exists a positive number $s>0$ such that

$$
|f(z)|=O\left(|z|^{-s}\right) \quad \text { as } \quad|z| \rightarrow \infty \text { for } z \in \Sigma_{\theta},
$$


and

$$
|f(z)|=O\left(|z|^{s}\right) \quad \text { as } \quad|z| \rightarrow 0 \text { for } z \in \Sigma_{\theta} .
$$

For any $\gamma \in(0, \pi)$, we let $\Gamma_{\gamma}$ be the boundary of $\Sigma_{\gamma}$, oriented counterclockwise. If $\theta \in(\gamma, \pi)$ and $f \in H_{0}^{\infty}\left(\Sigma_{\theta}\right)$, then (2.2) and (2.3) ensure that

$$
\int_{\Gamma_{\gamma}}|f(z)| \frac{d z}{z} \mid<\infty .
$$

Let $A$ be a sectorial operator of type $\omega$, and let $\theta \in(\omega, \pi)$. For any $f \in H_{0}^{\infty}\left(\Sigma_{\theta}\right)$, we set

$$
f(A)=\frac{1}{2 \pi i} \int_{\Gamma_{\gamma}} f(z) R(z, A) d z
$$

where $\gamma \in(\omega, \theta)$. It is clear from (2.4) and (2.1) that $f(A)$ is a well defined element of $B(X)$. Moreover its definition does not depend on the choice of $\gamma$, and the resulting mapping $f \mapsto f(A)$ is an algebra homomorphism from $H_{0}^{\infty}\left(\Sigma_{\theta}\right)$ into $B(X)$.

It is convenient to extend this functional calculus to bounded rational functions, as follows. For any $\lambda \in \mathbb{C} \backslash \overline{\Sigma_{\theta}}$, let $R_{\lambda} \in H^{\infty}\left(\Sigma_{\theta}\right)$ be defined by $R_{\lambda}(z)=(\lambda-z)^{-1}$. Then let

$$
\widetilde{H_{0}^{\infty}}\left(\Sigma_{\theta}\right)=H_{0}^{\infty}\left(\Sigma_{\theta}\right) \oplus \operatorname{Span}\left\{1, R_{-1}\right\} \subset H^{\infty}\left(\Sigma_{\theta}\right) .
$$

This is a subalgebra of $H^{\infty}\left(\Sigma_{\theta}\right)$. Next we let

$$
u_{A}: \widetilde{H_{0}^{\infty}}\left(\Sigma_{\theta}\right) \rightarrow B(X)
$$

be the linear mapping taking $f$ to $f(A)$ for any $f \in H_{0}^{\infty}\left(\Sigma_{\theta}\right)$, and taking 1 and $R_{-1}$ to $I_{X}$ and $R(-1, A)=-(1+A)^{-1}$ respectively. Then it is easy to check that $u_{A}$ is an algebra homomorphism. Moreover $R_{\lambda} \in \widetilde{H_{0}^{\infty}}\left(\Sigma_{\theta}\right)$ for any $\lambda \in \mathbb{C} \backslash \overline{\Sigma_{\theta}}$, and $u_{A}\left(R_{\lambda}\right)=R(\lambda, A)$. We call $u_{A}$ the holomorphic functional calculus of $A$ on $\widetilde{H_{0}^{\infty}}\left(\Sigma_{\theta}\right)$.

Definition 2.1. Let $A$ be a sectorial operator of type $\omega \in(0, \pi)$ on $X$ and let $\theta \in(\omega, \pi)$. We say that $A$ admits a bounded $H^{\infty}\left(\Sigma_{\theta}\right)$ functional calculus if there is a constant $C>0$ such that $\|f(A)\| \leq C\|f\|_{\infty, \theta}$ for any $f \in H_{0}^{\infty}\left(\Sigma_{\theta}\right)$.

Equivalently, $A$ admits a bounded $H^{\infty}\left(\Sigma_{\theta}\right)$ functional calculus if and only if the above homomorphism $u_{A}$ is continuous.

If $A$ is a sectorial operator of type $\omega \in(0, \pi)$ on $X$, and if $X$ is reflexive, then $A^{*}$ is a sectorial operator of type $\omega \in(0, \pi)$ as well. Furthermore given any $\theta>\omega$ and any function $f \in H_{0}^{\infty}\left(\Sigma_{\theta}\right)$, we have

$$
f(A)^{*}=\tilde{f}\left(A^{*}\right), \quad \text { where } \tilde{f}(z)=\overline{f(\bar{z})} \text { for any } z \in \Sigma_{\theta} .
$$

Consequently, $A^{*}$ admits a bounded $H^{\infty}\left(\Sigma_{\theta}\right)$ functional calculus if (and only if) $A$ does.

2.B. Semigroups and sectorial operators. We refer the reader to e.g. [17] for the necessary background on semigroup theory. Let $\left(T_{t}\right)_{t \geq 0}$ be a bounded $c_{0}$-semigroup on $X$, and let $-A$ denote its infinitesimal generator. Then $A$ is closed and densely defined, and we have $\sigma(A) \subset \overline{\Sigma_{\frac{\pi}{2}}}$. Moreover the Laplace formula says that whenever $\lambda \in \mathbb{C} \backslash \overline{\Sigma_{\frac{\pi}{2}}}$, we have

$$
R(\lambda, A)=-\int_{0}^{\infty} e^{\lambda t} T_{t} d t
$$


in the strong operator topology. Thus letting $C=\sup \left\{\left\|T_{t}\right\|: t \geq 0\right\}$, we see that for any $\lambda \in \mathbb{C} \backslash \overline{\Sigma_{\frac{\pi}{2}}}$, we have

$$
\|\lambda R(\lambda, A)\| \leq C\left(\frac{|\lambda|}{-\operatorname{Re}(\lambda)}\right) .
$$

It is easy to deduce from this estimate that $A$ is sectorial of type $\frac{\pi}{2}$.

We note in passing that there is a natural connection between the holomorphic functional calculus of $A$ and the so-called Phillips functional calculus of the semigroup $\left(T_{t}\right)_{t \geq 0}$. Indeed for any $b \in L^{1}\left(\mathbb{R}_{+} ; d t\right)$, let us denote by $\widehat{b}$ its Laplace transform defined on $\overline{\Sigma_{\frac{\pi}{2}}}$ by

$$
\widehat{b}(\lambda)=\int_{0}^{\infty} b(t) e^{-\lambda t} d t, \quad \lambda \in \overline{\Sigma_{\frac{\pi}{2}}} .
$$

Then for any $\theta \in\left(\frac{\pi}{2}, \pi\right)$ and for any $f \in H_{0}^{\infty}\left(\Sigma_{\theta}\right)$, there exists a (necessarily unique) function $b \in L^{1}\left(\mathbb{R}_{+} ; d t\right)$ such that

$$
f=\widehat{b} \text { on } \overline{\Sigma_{\frac{\pi}{2}}}, \quad \text { and } \quad f(A)=\int_{0}^{\infty} b(t) T_{t} d t .
$$

To prove this, take $\gamma \in\left(\frac{\pi}{2}, \theta\right)$ and define

$$
b(t)=-\int_{\Gamma_{\gamma}} f(z) e^{z t} d z, \quad t>0 .
$$

Then $b$ is integrable and using Fubini's Theorem, we obtain that for any $\lambda \in \overline{\Sigma_{\frac{\pi}{2}}}$,

$$
f(\lambda)=\int_{\Gamma_{\gamma}} \frac{f(z)}{z-\lambda} d z=-\int_{\Gamma_{\gamma}} f(z) \int_{0}^{\infty} e^{(z-\lambda) t} d t d z=\int_{0}^{\infty} b(t) e^{-\lambda t} d t=\widehat{b}(\lambda) .
$$

Likewiwe, using (2.5) and (2.7), one proves that $f(A) x=\int_{0}^{\infty} b(t) T_{t} x d t$ for any $x \in X$.

By definition (see e.g. [17, I.5]), a bounded analytic semigroup is a bounded $c_{0^{-}}$ semigroup $\left(T_{t}\right)_{t \geq 0}$ on $X$ such that there exist a positive angle $\alpha>0$ and a bounded analytic extension of $\left(T_{t}\right)_{t>0}$ on $\Sigma_{\alpha}$. That is, there exists a bounded family of operators $\left(T_{z}\right)_{z \in \Sigma_{\alpha}}$ extending $\left(T_{t}\right)_{t>0}$ and such that $z \mapsto T_{z}$ is analytic from $\Sigma_{\alpha}$ into $B(X)$. Note that such an extension necessarily satisfies $T_{z} T_{z^{\prime}}=T_{z+z^{\prime}}$ for any $z, z^{\prime} \in \Sigma_{\alpha}$. Generators of bounded analytic semigroups can be characterized as follows.

Proposition 2.2. Let $A$ be a linear operator on $X$. Then $-A$ is the infinitesimal generator of a bounded analytic semigroup if and only if $A$ is a sectorial operator of type $\omega<\frac{\pi}{2}$.

Proof. Assume that $-A$ generates a bounded analytic semigroup $\left(T_{z}\right)_{z \in \Sigma_{\alpha}}$ for some $\alpha$ in $\left(0, \frac{\pi}{2}\right)$. For any $\beta \in(-\alpha, \alpha),\left(T_{t e^{i \beta}}\right)_{t \geq 0}$ is a bounded $c_{0}$-semigroup whose generator is $-e^{i \beta} A$. Thus $e^{i \beta} A$ is sectorial of type $\frac{\pi}{2}$. Since this holds for any $\beta \in(-\alpha, \alpha)$, we deduce that $A$ is sectorial of type $\frac{\pi}{2}-\alpha$.

Assume conversely that $A$ is a sectorial operator of type $\omega \in\left(0, \frac{\pi}{2}\right)$. We fix two angles $\alpha>0$ and $\theta>\omega$ such that $\theta+\alpha<\frac{\pi}{2}$. Next for any $z \in \Sigma_{\alpha} \cup\{0\}$, we let $f_{z}$ be the analytic function defined by $f_{z}(\lambda)=e^{-z \lambda}$. Each $f_{z}$ belongs to $\widetilde{H_{0}^{\infty}}\left(\Sigma_{\theta}\right)$ and we may therefore define $T_{z}=u_{A}\left(f_{z}\right)$ for any $z \in \Sigma_{\alpha} \cup\{0\}$, where $u_{A}$ is the holomorphic functional calculus of $A$ on $\widetilde{H_{0}^{\infty}}\left(\Sigma_{\theta}\right)$. It is a simple matter to check that $z \mapsto T_{z}$ is analytic on $\Sigma_{\alpha}$. It therefore suffices to check that $\left(T_{t}\right)_{t \geq 1}$ is a bounded $c_{0}$-semigroup with generator $-A$, 
and that the family $\left(T_{z}\right)_{z \in \Sigma_{\alpha}}$ is bounded. This is left to the reader, see also [17, I.5.6] for details.

If $\left(T_{t}\right)_{t \geq 0}$ is a bounded $c_{0}$-semigroup on $X$ with generator $-A$, we will use the standard notation $T_{t}=e^{-t A}$ for any $t \geq 0$. If further $A$ is sectorial of type $\omega<\frac{\pi}{2}$, and if $z \in \Sigma_{\frac{\pi}{2}-\omega}$, then we will write $T_{z}=e^{-z A}$.

2.C. Sectorial operators with dense range. For any integer $n \geq 1$, let $f_{n}$ be the rational function defined by

$$
f_{n}(z)=\frac{n^{2} z}{(n+z)(1+n z)} .
$$

Let $A$ be a sectorial operator on $X$. Then the operators

$$
f_{n}(A)=\left[n A(1+n A)^{-1}\right]\left[n(n+A)^{-1}\right]
$$

are uniformly bounded. Moreover, if $x \in D(A)$, then we have

$$
n(n+A)^{-1} x-x=(n+A)^{-1} A x \rightarrow 0
$$

when $n \rightarrow \infty$, by (2.1). Likewise, $n A(1+n A)^{-1} x \rightarrow x$ for any $x \in R(A)$. These results imply the following approximation lemma.

Lemma 2.3. Let $A$ be a sectorial operator on $X$, and assume that $A$ has dense range. Let $\left(f_{n}\right)_{n \geq 1}$ be defined by (2.9). Then

$$
\sup _{n}\left\|f_{n}(A)\right\|<\infty \quad \text { and } \quad \lim _{n} f_{n}(A) x=x \text { for any } x \in X .
$$

We let $\varphi=f_{1}$, so that $\varphi(z)=z(1+z)^{-2}$ and $\varphi(A)=A(1+A)^{-2}$.

Proposition 2.4. Let $A$ be a sectorial operator with dense range on $X$. Then:

(1) $A$ is 1-1;

(2) $R(\varphi(A))=D(A) \cap R(A)$;

(3) The operator $\varphi(A)$ is 1-1 and has dense range.

Proof. If $x \in N(A)$, then $f_{n}(A) x=0$ for any $n \geq 1$. Hence $x=0$ by Lemma 2.3. The assertion (2) and the injectivity of $\varphi(A)$ are easy and left to the reader. To show that $D(A) \cap R(A)$ is dense, observe that $f_{n}(A) x$ belongs to that space for any $n \geq 1$ and apply Lemma 2.3.

Let $A$ be a sectorial operator of type $\omega \in(0, \pi)$, and assume that $A$ has dense range. Following $[42,11]$, one can construct a possibly unbounded operator $f(A)$ for any $f \in$ $H^{\infty}\left(\Sigma_{\theta}\right)$, provided that $\theta \in(\omega, \pi)$. Indeed for $f \in H^{\infty}\left(\Sigma_{\theta}\right)$, the product function $f \varphi$ belongs to $H_{0}^{\infty}\left(\Sigma_{\theta}\right)$, hence we may define $(f \varphi)(A)$ by $(2.5)$. Then using Proposition 2.4 we set

$$
f(A)=\varphi(A)^{-1}(f \varphi)(A)
$$

with domain given by

$$
D(f(A))=\{x \in X:[(f \varphi)(A)](x) \in D(A) \cap R(A)\} .
$$

This domain contains $D(A) \cap R(A)$ and is therefore dense. Furthermore the operator $f(A)$ is closed because $\varphi(A)$ is bounded. Thus $f(A)$ is bounded if and only if $D(f(A))=X$. 
Using Lemma 2.3 and Proposition 2.4, one obtains the following fundamental result, which shows the relevance of the preceding definitions.

Theorem 2.5 (McIntosh). Let $A$ be a sectorial operator of type $\omega \in(0, \pi)$ on $X$, and assume that $A$ has dense range. Let $\theta \in(\omega, \pi)$ be an angle. Then $A$ has a bounded $H^{\infty}\left(\Sigma_{\theta}\right)$ functional calculus (in the sense of Definition 2.1) if and only if $f(A)$ is a bounded operator for any $f \in H^{\infty}\left(\Sigma_{\theta}\right)$. In this case the mapping $f \mapsto f(A)$ is a bounded homomorphism from $H^{\infty}\left(\Sigma_{\theta}\right)$ into $B(X)$, which extends $u_{A}$.

REMARK 2.6. Let $A$ be a sectorial operator on $X$. If $X$ is reflexive, then there is a direct sum decomposition

$$
X=N(A) \oplus \overline{R(A)},
$$

and the restriction of $A$ to $\overline{R(A)}$ is a sectorial operator with dense range (see [11, Theorem $3.8]$ ). Thus $A$ is $1-1$ if and only if $A$ has dense range, and one can easily reduce to this case by changing $A$ into its restriction to $\overline{R(A)}$.

2.D. Rademacher boundedness. In this subsection, we introduce the concepts of $R$ boundedness (see $[5,8]$ ) and $R$-sectoriality (see [56], [57], [29]), which now play a fundamental role for the study of sectorial operators and $H^{\infty}$ functional calculus.

Consider a Rademacher sequence $\left(\varepsilon_{k}\right)_{k \geq 1}$ on a probability space $(\Sigma, \mathbb{P})$, that is, a sequence of pairwise independent random variables on $\Sigma$ such that $\mathbb{P}\left(\varepsilon_{k}=1\right)=\mathbb{P}\left(\varepsilon_{k}=\right.$ $-1)=\frac{1}{2}$ for any $k \geq 1$. For any finite family $x_{1}, \ldots, x_{n}$ in a Banach space $X$, we let

$$
\left\|\sum_{k=1}^{n} \varepsilon_{k} x_{k}\right\|_{\operatorname{Rad}(X)}=\left(\int_{\Sigma}\left\|\sum_{k=1}^{n} \varepsilon_{k}(s) x_{k}\right\|_{X}^{2} d \mathbb{P}(s)\right)^{\frac{1}{2}} \text {. }
$$

By definition, a set $\mathcal{T} \subset B(X)$ is $R$-bounded if there is a constant $C \geq 0$ such that for any finite families $T_{1}, \ldots, T_{n}$ in $\mathcal{T}$, and any $x_{1}, \ldots, x_{n}$ in $X$, we have

$$
\left\|\sum_{k=1}^{n} \varepsilon_{k} T_{k}\left(x_{k}\right)\right\|_{\operatorname{Rad}(X)} \leq C\left\|\sum_{k=1}^{n} \varepsilon_{k} x_{k}\right\|_{\operatorname{Rad}(X)} .
$$

If $H$ is a Hilbert space, then for any $x_{1}, \ldots, x_{n}$ in $H$ we have

$$
\left\|\sum_{k=1}^{n} \varepsilon_{k} x_{k}\right\|_{\operatorname{Rad}(H)}=\left(\sum_{k=1}^{n}\left\|x_{k}\right\|^{2}\right)^{\frac{1}{2}} .
$$

Hence any bounded set $\mathcal{T} \subset B(H)$ is automatically $R$-bounded. More generally, any bounded set $\mathcal{T} \subset B(X)$ is automatically $R$-bounded if $X$ is isomorphic to a Hilbert space. Conversely, if $X$ is not isomorphic to a Hilbert space, then there exist bounded subsets of $B(X)$ which are not $R$-bounded (see e.g. [2, Proposition 1.13]).

If $A$ is a sectorial operator on $X$ and $\omega \in(0, \pi)$ is an angle, we say that $A$ is $R$-sectorial of $R$-type $\omega$ if for any $\theta \in(\omega, \pi)$, the set

$$
\left\{\lambda R(\lambda, A): \lambda \in \mathbb{C} \backslash \overline{\Sigma_{\theta}}\right\} \subset B(X)
$$

is $R$-bounded. The following result due to Kalton and Weis is a key connection between $H^{\infty}$ calculus and $R$-boundedness. 
THEOREM 2.7 (Kalton-Weis [29]). Let $A$ be a sectorial operator on $X$, and assume that $X$ has property $(\Delta)$. If $A$ has a bounded $H^{\infty}\left(\Sigma_{\theta}\right)$ functional calculus, then $A$ is $R$-sectorial of $R$-type $\theta$.

A few words on $(\Delta)$ and related Banach space geometry is in order. Let $\left(\varepsilon_{k}\right)_{k \geq 1}$ and $\left(\varepsilon_{j}^{\prime}\right)_{j \geq 1}$ be two mutually independent Rademacher sequences on $(\Sigma, \mathbb{P})$. Following [29], we say that a Banach space $X$ has property $(\Delta)$ if there is a constant $K \geq 0$ such that

$$
\int_{\Sigma}\left\|\sum_{1 \leq j \leq k \leq n} \varepsilon_{j}^{\prime}(s) \varepsilon_{k}(s) x_{j k}\right\|_{X}^{2} d \mathbb{P}(s) \leq K^{2} \int_{\Sigma}\left\|\sum_{j, k=1}^{n} \varepsilon_{j}^{\prime}(s) \varepsilon_{k}(s) x_{j k}\right\|_{X}^{2} d \mathbb{P}(s)
$$

for any $n \geq 1$ and any $x_{j k}$ in $X$.

We recall that a Banach space $X$ is UMD if for some $1<p<\infty$ (equivalently, for all $1<p<\infty)$, the Hilbert transform is bounded on $L^{p}(\mathbb{R} ; X)$. UMD Banach spaces include Hilbert spaces, as well as $L^{p}$-spaces for any $1<p<\infty$. Indeed, if $\Omega$ is any measure space and $Y$ is a UMD Banach space, then $X=L^{p}(\Omega ; Y)$ is UMD for any $1<p<\infty$. Moreover subspaces of UMD Banach spaces are UMD. We refer the reader to [7] and the references therein for more information on this topic.

It is shown in [29] that any UMD Banach space has property $(\Delta)$. In fact, it is shown in [29] that a Banach space $X$ has property $(\Delta)$ provided that it has the so-called analytic UMD property (see also [20]). Analytic UMD Banach spaces include UMD spaces, as well as $L^{1}$-spaces and their subspaces (see $[16,7]$ ). Thus Theorem 2.7 applies to any subspace of $L^{p}$, for any $1 \leq p<\infty$.

We wish to mention that the notion of $R$-sectoriality on non Hilbertian Banach spaces is closely related to maximal $L^{p}$-regularity. Namely, it was proved in [28] and [56] that if $A$ is a sectorial operator of type $<\frac{\pi}{2}$ on a Banach space $X$ with maximal $L^{p}$-regularity, then $A$ is $R$-sectorial of $R$-type $<\frac{\pi}{2}$. Thus the counterexamples to maximal $L^{p}$-regularity obtained by Kalton-Lancien in [28] show that when $p \neq 2$, there exist sectorial operators on $L^{p}$-spaces which are not $R$-sectorial. Conversely, it was proved in [56] that if $X$ is a UMD Banach space, and $A$ is $R$-sectorial of $R$-type $<\frac{\pi}{2}$ on $X$, then $A$ has maximal $L^{p}$-regularity.

\section{Square functions on Hilbert space}

3.A. Square functions and their equivalence. Square functions for sectorial operators on Hilbert space were introduced by McIntosh in [42], with important developments in [43] (see also [1, 4]). After introducing them, we will focus on their equivalence and their connections with $H^{\infty}$ functional calculus.

Throughout this section, we let $H$ be a Hilbert space and we let $A$ be a sectorial operator of type $\omega \in(0, \pi)$ on $H$. We define

$$
H_{0}^{\infty}\left(\Sigma_{\omega+}\right)=\cup_{\theta>\omega} H_{0}^{\infty}\left(\Sigma_{\theta}\right) .
$$

Then for any $F \in H_{0}^{\infty}\left(\Sigma_{\omega+}\right)$, we set

$$
\|x\|_{F}=\left(\int_{0}^{\infty}\|F(t A) x\|^{2} \frac{d t}{t}\right)^{1 / 2}, \quad x \in H .
$$


In the above definition $F(t A)$ means $F_{t}(A)$, where $F_{t}(z)=\varphi(t z)$. Using Lebesgue's continuity Theorem, it is easy to check that $t \mapsto F(t A)$ is continuous hence $\|x\|_{F}$ is well defined for any $x \in X$. Note however that we may have $\|x\|_{F}=\infty$. We call \|\|$_{F}$ a square function associated with $A$.

Theorem 3.1 (McIntosh-Yagi [43]). Let $A$ be a sectorial operator of type $\omega \in(0, \pi)$ on some Hilbert space $H$, and assume that $A$ is 1-1. Let $F$ and $G$ be nonzero functions in $H_{0}^{\infty}\left(\Sigma_{\theta}\right)$, for some $\theta>\omega$.

(1) There is a constant $K>0$ such that for any $f \in H^{\infty}\left(\Sigma_{\theta}\right)$,

$$
\left(\int_{0}^{\infty}\|g(A) F(t A) x\|^{2} \frac{d t}{t}\right)^{\frac{1}{2}} \leq K\|g\|_{\infty, \theta}\|x\|_{G}, \quad x \in H .
$$

(2) There is a constant $K>0$ such that

$$
K^{-1}\|x\|_{G} \leq\|x\|_{F} \leq K\|x\|_{G}, \quad x \in H
$$

Proof. For simplicity we will prove (2) only. The proof of (1) is quite similar (see [43], [1], or [35] for details). Let $\theta \in(\omega, \pi)$, and let $F$ and $G$ be two nonzero functions belonging to $H_{0}^{\infty}\left(\Sigma_{\theta}\right)$. We introduce an auxiliary function $\varphi \in H_{0}^{\infty}\left(\Sigma_{\theta}\right)$ such that

$$
\int_{0}^{\infty} \varphi(t) G(t) \frac{d t}{t}=1
$$

Then we have $\int_{0}^{\infty} \varphi(t z) G(t z) \frac{d t}{t}=1$. for any $z \in \Sigma_{\theta}$. Indeed it is clear when $z$ is a positive real number, and hence for general $z$ by analytic continuation. By Fubini's Theorem and (2.5), this implies that

$$
f(A)=\int_{0}^{\infty} \varphi(t A) G(t A) f(A) \frac{d t}{t}
$$

for any $f \in H_{0}^{\infty}\left(\Sigma_{\theta}\right)$, the latter integral being absolutely convergent.

We let $\gamma \in(\omega, \theta)$ be an intermediate angle, and we let $\Gamma=\Gamma_{\gamma}$ be the corresponding contour used in (2.5). Next we consider the two measure spaces $\Omega_{0}=\left(\mathbb{R}_{+}^{*} ; \frac{d t}{t}\right)$ and $\Omega=\left(\Gamma,\left|\frac{d z}{z}\right|\right)$. We may define a bounded operator $\sigma: L^{2}\left(\Omega_{0}\right) \rightarrow L^{2}(\Omega)$ by letting

$$
[\sigma(a)](z)=\int_{0}^{\infty} \varphi(t z) a(t) \frac{d t}{t}, \quad a \in L^{2}\left(\Omega_{0}\right), z \in \Omega .
$$

To check this, we first observe that

$$
K=\sup _{t>0} \int_{\Gamma}|\varphi(t z)|\left|\frac{d z}{z}\right|<\infty \quad \text { and } \quad K^{\prime}=\sup _{z \in \Gamma} \int_{0}^{\infty}|\varphi(t z)| \frac{d t}{t}<\infty .
$$

Indeed, changing $z$ into $t z$ does not change $\int_{\Gamma}|\varphi(t z)|\left|\frac{d z}{z}\right|$ hence $K=\int_{\Gamma}|\varphi(z)|\left|\frac{d z}{z}\right|$, which is finite by (2.4). On the other hand, for any $z \in \Gamma \backslash\{0\}$ we have

$$
\int_{0}^{\infty}|\varphi(t z)| \frac{d t}{t}+\int_{0}^{\infty}|\varphi(t \bar{z})| \frac{d t}{t}=\int_{\Gamma}|\varphi(\lambda)| \frac{d \lambda}{\lambda} \mid
$$

hence $K^{\prime} \leq K<\infty$. 
We let $a$ be an arbitrary element of $L^{2}\left(\Omega_{0}\right)$. Then by Cauchy-Schwarz and (3.3), we have

$$
\begin{aligned}
\int_{\Gamma}|[\sigma(a)](z)|^{2}\left|\frac{d z}{z}\right| & \leq \int_{\Gamma}\left(\int_{0}^{\infty}|\varphi(t z)| \frac{d t}{t}\right)\left(\int_{0}^{\infty}|\varphi(t z)||a(t)|^{2} \frac{d t}{t}\right)\left|\frac{d z}{z}\right| \\
& \leq K^{\prime} \int_{\Gamma}\left(\int_{0}^{\infty}|\varphi(t z)||a(t)|^{2} \frac{d t}{t}\right)\left|\frac{d z}{z}\right| \leq K K^{\prime} \int_{0}^{\infty}|a(t)|^{2} \frac{d t}{t} .
\end{aligned}
$$

This shows that $\left\|\sigma: L^{2}\left(\Omega_{0}\right) \rightarrow L^{2}(\Omega)\right\| \leq \sqrt{K K^{\prime}}$. Since $H$ is a Hilbert space, the tensor extension

$$
\sigma \otimes I_{H}: L^{2}\left(\Omega_{0}\right) \otimes H \rightarrow L^{2}(\Omega) \otimes H
$$

extends to a bounded operator $S: L^{2}\left(\Omega_{0} ; H\right) \rightarrow L^{2}(\Omega ; H)$, with $\|S\| \leq \sqrt{K K^{\prime}}$.

Let $x \in H$ such that $\|x\|_{G}<\infty$, and let $u \in L^{2}\left(\Omega_{0} ; H\right)$ be defined by $u(t)=G(t A) x$. For any $z \in \Gamma$, let

$$
w(z)=\int_{0}^{\infty} \varphi(t z) G(t A) x \frac{d t}{t}=\int_{0}^{\infty} \varphi(t z) u(t) \frac{d t}{t} .
$$

Then $w=S(u) \in L^{2}(\Omega ; H)$, and letting $C=\|S\|$, we obtain that

$$
\int_{\Gamma}\|w(z)\|^{2}\left|\frac{d z}{z}\right| \leq C^{2}\|x\|_{G}^{2}
$$

Next we define

$$
w^{\prime}(z)=z R(z, A) f(A) w(z) \quad \text { and } \quad v(s)=\frac{1}{2 \pi i} \int_{0}^{\infty} F(s z) w^{\prime}(z) \frac{d z}{z}
$$

for $z \in \Omega$ and $s \in \Omega_{0}$. Using (3.2) and Fubini's Theorem, it is fairly easy to check that

$$
v(s)=f(A) F(s A) x, \quad s>0 .
$$

Letting $C^{\prime}=\sup _{z \in \Gamma}\{\|z R(z, A)\|\}$, we see that

$$
\int_{\Gamma}\left\|w^{\prime}(z)\right\|^{2}\left|\frac{d z}{z}\right| \leq C^{\prime 2}\|f(A)\|^{2} \int_{\Gamma}\|w(z)\|^{2}\left|\frac{d z}{z}\right| .
$$

Moreover, arguing as in the proof of (3.4), we can find a constant $C^{\prime \prime}$ such that

$$
\int_{0}^{\infty}\|v(s)\|^{2} \frac{d t}{t} \leq C^{\prime 2} \int_{\Gamma}\left\|w^{\prime}(z)\right\|^{2}\left|\frac{d z}{z}\right| .
$$

Altogether, we finally obtain that

$$
\|f(A) x\|_{F}=\|v\|_{L^{2}\left(\Omega_{0} ; H\right)} \leq C C^{\prime} C^{\prime \prime}\|f(A)\|\|x\|_{G} .
$$

Now using Lemma 2.3 and applying the above estimate with $f_{n}$ instead of $f$, we find that \|\|$_{G}$ dominates \|\|$_{F}$. By symmetry, \|\|$_{G}$ and \|\|$_{F}$ are therefore equivalent.

3.B. Square functions and $H^{\infty}$ functional calculus. Suppose that $A$ is a sectorial operator of type $\omega$ on $H$, and let $G \in H_{0}^{\infty}\left(\Sigma_{\omega+}\right)$. We will denote by \|\|$_{G}^{*}$ the square function for $G$ associated with the adjoint operator $A^{*}$. Namely,

$$
\|y\|_{G}^{*}=\left(\int_{0}^{\infty}\left\|G\left(t A^{*}\right) y\right\|^{2} \frac{d t}{t}\right)^{1 / 2}, \quad y \in H .
$$


TheOrem 3.2 (McIntosh [42]). Let $A$ be a 1-1 sectorial operator of type $\omega \in(0, \pi)$ on some Hilbert space $H$, and let $\theta \in(\omega, \pi)$. The following assertions are equivalent.

(i) A has a bounded $H^{\infty}\left(\Sigma_{\theta}\right)$ functional calculus.

(ii) For some (equivalently, for any) pair $(F, G)$ of nonzero functions in $H_{0}^{\infty}\left(\Sigma_{\omega+}\right)$, there is a constant $K>0$ such that

$$
\|x\|_{F} \leq K\|x\| \quad \text { and } \quad\|y\|_{G}^{*} \leq K\|y\|
$$

for any $x, y \in H$.

(iii) For some (equivalently, for any) nonzero function $F \in H_{0}^{\infty}\left(\Sigma_{\omega+}\right)$, there is a constant $K>0$ such that

$$
K^{-1}\|x\| \leq\|x\|_{F} \leq K\|x\|, \quad x \in H .
$$

Proof. (i) $\Rightarrow$ (ii): We assume that $A$ admits a bounded $H^{\infty}\left(\Sigma_{\theta}\right)$ functional calculus, and we let $F \in H_{0}^{\infty}\left(\Sigma_{\theta}\right)$. By [29, Lemma 4.1] and its proof, there exists a constant $K>0$ such that whenever $\left(\alpha_{k}\right)_{k \in \mathbb{Z}}$ is a finite sequence of complex numbers and $t>0$ is a positive real number, we have

$$
\left\|\sum_{k} \alpha_{k} F\left(t 2^{k} A\right)\right\| \leq K \max _{k}\left|\alpha_{k}\right|
$$

For any $x \in X$, we have

$$
\int_{0}^{\infty}\|F(t A) x\|^{2} \frac{d t}{t}=\sum_{k=-\infty}^{\infty} \int_{2^{k}}^{2^{k+1}}\|F(t A) x\|^{2} \frac{d t}{t}=\int_{1}^{2} \sum_{k=-\infty}^{\infty}\left\|F\left(t 2^{k} A\right) x\right\|^{2} \frac{d t}{t}
$$

by changing $t$ into $t 2^{-k}$. Then using (2.10), we have for any $n \geq 1$ and any $t \in[1,2]$,

$$
\begin{aligned}
\sum_{k=-n}^{k=n}\left\|F\left(t 2^{k} A\right) x\right\|^{2} & =\left\|\sum_{k=-n}^{n} \varepsilon_{k} F\left(t 2^{k} A\right) x\right\|_{\operatorname{Rad}(H)}^{2} \\
& \leq\left\|\sum_{k=-n}^{n} \varepsilon_{k} F\left(t 2^{k} A\right)\right\|_{\operatorname{Rad}(B(H))}^{2}\|x\|^{2} \leq K^{2}\|x\|^{2}
\end{aligned}
$$

by (3.5). We therefore deduce that $\|x\|_{F}^{2} \leq K^{2}\|x\|^{2}$. This proves the first half of (ii). Changing $A$ into $A^{*}$, we obtain the second half.

(iii) $\Rightarrow$ (i): This is a straightforward consequence of Theorem 3.1.

(ii) $\Rightarrow$ (iii): Let $\theta>\omega$ be an angle. Let $(F, G)$ be two functions in $H_{0}^{\infty}\left(\Sigma_{\theta}\right)$, such that

$$
\int_{0}^{\infty} F(t) \widetilde{G}(t) \frac{d t}{t}=1
$$

Here $\widetilde{G}$ is defined as in (2.6). Arguing as in the proof of Theorem 3.1, we obtain that for any $f \in H_{0}^{\infty}\left(\Sigma_{\theta}\right)$, we have

$$
f(A)=\int_{0}^{\infty} f(A) F(t A) G\left(t A^{*}\right)^{*} \frac{d t}{t} .
$$

This implies that for any $x, y \in H$, we have

$$
\langle f(A) x, y\rangle=\int_{0}^{\infty}\left\langle f(A) F(t A) x, G\left(t A^{*}\right) y\right\rangle \frac{d t}{t} .
$$


Using Cauchy-Schwarz, we deduce that

$$
|\langle f(A) x, y\rangle| \leq\|f(A)\|\|x\|_{F}\|y\|_{G}^{*} .
$$

Applying Lemma 2.3 and the above estimate with $f_{n}$ instead of $f$, we deduce that for a certain constant $K>0$, we have

$$
|\langle x, y\rangle| \leq K\|x\|_{F}\|y\|_{G}^{*}, \quad x, y \in H .
$$

By assumption, $\|y\|_{G}^{*}$ is dominated by $\|y\|$. With $y=x$, we deduce that $\|x\| \leq K^{\prime}\|x\|_{F}$ for a certain constant $K^{\prime}$ not depending on $x$.

REMARK 3.3. Let $A$ be a sectorial operator on $H$, with type $\omega$.

(1) As a consequence of Theorem 3.2, we see that $A$ has a bounded $H^{\infty}\left(\Sigma_{\theta}\right)$ functional calculus for all $\theta \in(\omega, \pi)$ if it has a bounded $H^{\infty}\left(\Sigma_{\theta}\right)$ functional calculus for some $\theta \in(\omega, \pi)$. We will simply say that $A$ has a bounded $H^{\infty}$ functional calculus in that case.

(2) We say that $A$ satisfies a square function estimate if for some (equivalently, for all) $F \in H_{0}^{\infty}\left(\Sigma_{\omega+}\right)$, there is a constant $K>0$ such that $\|x\|_{F} \leq K\|x\|$ for any $x \in H$. By Theorem 3.2 (and Remark 2.6), $A$ has a bounded $H^{\infty}$ functional calculus if and only if both $A$ and $A^{*}$ satisfy a square function estimate. In [34], an example is given of an operator $A$ which satisfies a square function estimate, although it does not have a bounded $H^{\infty}$ functional calculus.

(3) Assume that $A$ is $1-1$, and let $F \in H_{0}^{\infty}\left(\Sigma_{\omega+}\right) \backslash\{0\}$. Then the set $\mathcal{H}_{F}$ of all $x \in H$ such that $\|x\|_{F}<\infty$ is a subspace of $H$, and \|\|$_{F}$ is a Hilbertian norm on $\mathcal{H}_{F}$. It is not hard to check that $\|x\|_{F}<\infty$ for any $x \in D(A) \cap R(A)$, hence $\mathcal{H}_{F}$ is a dense subset of $H$, by Proposition 2.4. Let $H_{F}$ be the completion of $\left(\mathcal{H}_{F},\|\|_{F}\right)$. By Theorem 3.1, the identity mapping on $D(A) \cap R(A)$ induces an isomorphism $H_{F} \approx H_{G}$ for any pair $(F, G)$ of nonzero functions in $H_{0}^{\infty}\left(\Sigma_{\omega+}\right)$. On the other hand, Theorem 3.2 implies that $H_{F} \approx H$ if and only if $A$ has an $H^{\infty}$ functional calculus. We refer the reader to [4] for more on $H_{F}$ and a description of that space in terms of complex interpolation.

4. Two applications. We present two applications of square functions to operator theoretical problems for bounded analytic semigroups. We will freely use the notation and results discussed in 2.B. Both applications will use the following simple observation. Let $F_{0}$ be defined by

$$
F_{0}(z)=z^{\frac{1}{2}} e^{-z}
$$

Clearly $F_{0} \in H_{0}^{\infty}\left(\Sigma_{\theta}\right)$ for any $\theta<\frac{\pi}{2}$.

LEMmA 4.1. Let $A$ be a sectorial operator of type $<\frac{\pi}{2}$ on some Hilbert space $H$, and let $\left(T_{t}\right)_{t \geq 0}$ be the bounded analytic semigroup on $H$ generated by $-A$.

(1) For any $x \in H$, we have

$$
\|x\|_{F_{0}}^{2}=\int_{0}^{\infty}\left\|A^{\frac{1}{2}} T_{t} x\right\|^{2} d t .
$$

(2) For any $x \in H$ and any $t>0$, we have

$$
\left\|T_{t} x\right\|_{F_{0}} \leq\|x\|_{F_{0}} .
$$


Proof. (1) is clear from the definitions. For (2), consider $x \in H$ and $t>0$. Then by (1),

$$
\begin{aligned}
\left\|T_{t} x\right\|_{F_{0}}^{2} & =\int_{0}^{\infty}\left\|A^{\frac{1}{2}} T_{s} T_{t} x\right\|^{2} d s=\int_{0}^{\infty}\left\|A^{\frac{1}{2}} T_{s+t} x\right\|^{2} d s \\
& =\int_{t}^{\infty}\left\|A^{\frac{1}{2}} T_{s} x\right\|^{2} d s \leq \int_{0}^{\infty}\left\|A^{\frac{1}{2}} T_{s} x\right\|^{2} d s=\|x\|_{F_{0}}^{2} .
\end{aligned}
$$

4.A. Bounded analytic semigroups similar to contractions. Let $H$ be a Hilbert space. We say that an operator $B \in B(H)$ is similar to a contraction if there exists an invertible operator $S \in B(H)$ such that $\left\|S^{-1} B S\right\| \leq 1$. The question of determining operators similar to contractions has attracted much attention in the last two decades. A key result for this topic is the von Neumann inequality which asserts that if $B \in B(H)$ is a contraction, then

$$
\|\varphi(B)\| \leq \sup \{|\varphi(w)|: w \in \mathbb{D}\}
$$

for any $\varphi$ in the disc algebra $A(\mathbb{D})$. Here $\mathbb{D}=\{w \in \mathbb{C}:|w|<1\}$ is the unit disc of $\mathbb{C}$. Thus if $B$ is similar to a contraction, then there is a constant $K>0$ such that

$$
\|\varphi(B)\| \leq K \sup \{|\varphi(w)|: w \in \mathbb{D}\}, \quad \varphi \in A(\mathbb{D}) .
$$

An operator $B$ satisfying (4.2) is called polynomially bounded. Solving a famous conjecture due to Halmos, Pisier proved in [47] that there exist polynomially bounded operators not similar to contractions. Paulsen proved in [45] that $B \in B(H)$ is similar to a contraction if and only if it is completely polynomially bounded, a stronger condition meaning that (4.2) remains valid with a constant $K$ independent of $n$ when $\varphi$ belongs to the matrix disc algebra $M_{n}(A(\mathbb{D}))$. We refer to $[46,48]$ and the references therein for more details and various results on similarity problems.

We say that a bounded $c_{0}$-semigroup $\left(T_{t}\right)_{t \geq 0}$ on $H$ is similar to a contraction if there is an invertible operator $S \in B(H)$ such that $\left\|S^{-1} T_{t} S\right\| \leq 1$ for any $t \geq 0$. If $-A$ is the infinitesimal generator of $\left(T_{t}\right)_{t \geq 0}$, this is equivalent to saying that $S^{-1} A S$ is maximal accretive. Let

$$
B=(A-1)(A+1)^{-1}
$$

be the cogenerator of $A$ (see e.g. [44]). Then $B$ is a contraction if and only if $\left(T_{t}\right)_{t \geq 0}$ is a contraction semigroup, and $B$ is similar to a contraction if and only if $\left(T_{t}\right)_{t \geq 0}$ is similar to contraction. Thus the problems of characterizing operators similar to contractions and semigroups similar to contractions are essentially equivalent. In this part, we want to show how square functions can be used to deal with the specific class of bounded analytic semigroups. The following result is due to the author $[36,37]$ and independently to Franks [15] and Callier-Grabowsky (in the case when $A$ is invertible, [18]).

Theorem 4.2. Let $A$ be a sectorial operator of type $\omega<\frac{\pi}{2}$ on $H$, and let $\left(T_{t}\right)_{t \geq 0}$ be generated by $-A$. Let $0<\alpha<\frac{\pi}{2}-\omega$ be an angle. The following assertions are equivalent.

(i) The operator $A$ has a bounded $H^{\infty}$ functional calculus.

(ii) There is an invertible operator $S \in B(H)$ such that $\left\|S^{-1} e^{-z A} S\right\| \leq 1$ for any $z \in \Sigma_{\alpha}$.

(iii) The semigroup $\left(T_{t}\right)_{t \geq 0}$ is similar to a contraction. 
Proof. (ii) $\Rightarrow$ (iii) is obvious.

(iii) $\Rightarrow$ (i): We fix an angle $\theta>\frac{\pi}{2}$, and we let $B$ be the cogenerator of $A$. According to our previous discussion, $B$ is polynomially bounded, that is, it satisfies (4.2). We set $\tau(w)=(1+w)(1-w)^{-1}$ for any $w \neq 1$. Let $f \in H_{0}^{\infty}\left(\Sigma_{\theta}\right)$ and let $\varphi: \mathbb{D} \rightarrow \mathbb{C}$ be defined by $\varphi=f \circ \tau$. Since $\tau$ induces a conformal mapping from $\mathbb{D}$ onto $\Sigma_{\frac{\pi}{2}}$, the supremum of $|\varphi|$ on $\mathbb{D}$ is equal to $\|f\|_{\infty, \frac{\pi}{2}}$. Furthermore, $\varphi$ belongs to the disc algebra and $\varphi(B)=f(A)$. Hence

$$
\|f(A)\| \leq K\|f\|_{\infty, \frac{\pi}{2}} .
$$

This shows that $A$ has a bounded $H^{\infty}$ functional calculus.

(i) $\Rightarrow$ (ii): Using Remark 2.6, we may assume that $A$ is 1 -1. It suffices to show that there exists an equivalent Hilbertian norm $|\|\||$ on $H$ such that $\left|\left\|e^{-z A} x\right\|\right| \leq|\|x\||$ for any $z \in \Sigma_{\alpha}$ and any $x \in H$. (Indeed in that case, the identity mapping $S$ from $(H,|\||\||)$ onto $(H,\|\|)$ is an isomorphism and $S^{-1} e^{-z A} S$ is a contraction.) By Theorem 3.2 (see also Remark 3.3), \|\|$_{F}$ is an equivalent Hilbertian norm on $H$ for any nonzero $F \in H_{0}^{\infty}\left(\Sigma_{\omega+}\right)$. Thus we only need to find such an $F$ for which

$$
\left\|e^{-z A} x\right\|_{F} \leq\|x\|_{F}, \quad z \in \Sigma_{\alpha}, x \in H .
$$

We fix two numbers $\varepsilon>0$ and $\nu>\frac{2 \omega}{\pi}$ such that

$$
\alpha+\nu\left(\frac{\pi}{2}+\varepsilon\right)<\frac{\pi}{2} .
$$

We let $C=A^{\frac{1}{\nu}}$, which is a sectorial operator of type $\frac{\omega}{\nu}<\frac{\pi}{2}$. We set

$$
F(z)=F_{0}\left(z^{\frac{1}{\nu}}\right)=z^{\frac{1}{2 \nu}} e^{-z^{\frac{1}{\nu}}},
$$

where $F_{0}$ is the special function considered in Lemma 4.1. Since $\frac{\omega}{\nu}<\frac{\pi}{2}$, the function $F$ belongs to $H_{0}^{\infty}\left(\Sigma_{\omega+}\right)$. For any $x \in H$, we have

$$
\|x\|_{F}^{2}=\int_{0}^{\infty}\left\|t^{\frac{1}{2 \nu}} C^{\frac{1}{2}} e^{-t^{\frac{1}{\nu}} C} x\right\|^{2} \frac{d t}{t}=\nu \int_{0}^{\infty}\left\|C^{\frac{1}{2}} e^{-s C} x\right\|^{2} d s
$$

by letting $s=t^{\frac{1}{\nu}}$. Applying Lemma 4.1 to $C$, we deduce that

$$
\left\|e^{-t C} x\right\|_{F} \leq\|x\|_{F}, \quad x \in H, t>0 .
$$

Thus $\left(e^{-t C}\right)_{t \geq 0}$ is a contraction semigroup on the Hilbert space $H_{F}=\left(H,\|\|_{F}\right)$. Applying von Neumann's inequality as in the proof of '(iii) $\Rightarrow$ (i)' above, we deduce that for any $\theta>\frac{\pi}{2}$ and any $f \in \widetilde{H_{0}^{\infty}}\left(\Sigma_{\theta}\right)$, we have

$$
\|f(C) x\|_{F} \leq\|f\|_{\infty, \frac{\pi}{2}}\|x\|_{F}, \quad x \in H .
$$

We fix a complex number $z \in \Sigma_{\alpha}$, and we define

$$
f(\lambda)=e^{-z \lambda^{\nu}}, \quad \lambda \in \mathbb{C} .
$$

Let $\theta=\frac{\pi}{2}+\varepsilon$ and recall that $\alpha+\nu \theta<\frac{\pi}{2}$. This angle condition ensures that $f \in \widetilde{H_{0}^{\infty}}\left(\Sigma_{\theta}\right)$, and moreover we have $\|f\|_{\infty, \frac{\pi}{2}}=1$. Hence $\|f(C) x\|_{F} \leq\|x\|_{F}$ for any $x \in H$ by (4.4). Since $f(C)=e^{-z A}$, this yields (4.3).

REMARK 4.3. Let $\left(T_{t}\right)_{t \geq 0}$ be a bounded $c_{0}$-semigroup on $H$, and assume that its negative generator $A$ is $1-1$. We do not assume the semigroup to be analytic in this subsection. For any real number $s$, let $A^{i s}$ be the corresponding imaginary power of $A$. Using Subsection 
2.C, these may be defined as $A^{i s}=\psi_{s}(A)$, where $\psi_{s}(z)=z^{i s}$. Using von Neumann's inequality as in the proof of Theorem 4.2, one obtains that if $\left(T_{t}\right)_{t \geq 0}$ is a contraction semigroup, then $\left\|A^{i s}\right\| \leq e^{\frac{\pi}{2}|s|}$ for any $s \in \mathbb{R}$. It is shown in [38] that conversely, if $\left\|A^{i s}\right\| \leq e^{\frac{\pi}{2}|s|}$ for any $s \in \mathbb{R}$, then $\left(T_{t}\right)_{t \geq 0}$ is similar to a contraction semigroup.

4.B. An application to control theory. Let $\left(T_{t}\right)_{t \geq 0}$ be a bounded $c_{0}$-semigroup on some Hilbert space $H$, and let $-A$ denote its generator. Let $K$ be another Hilbert space and let $C: D(A) \rightarrow K$ be a linear operator defined on the domain of $A$. We assume that $C$ is continuous with respect to the graph norm of $D(A)$. By definition, $C$ is admissible for $A$ if there is a constant $M>0$ such that

$$
\int_{0}^{\infty}\left\|C T_{t} x\right\|^{2} d t \leq M^{2}\|x\|^{2}, \quad x \in D(A) .
$$

The problem of characterizing admissibility arises from control theory. For a wide information on this topic, we refer the reader to the excellent survey [23] and the references therein. A key observation due to Weiss [55] is that if $C$ is admissible for $A$, then there is a constant $D>0$ such that

$$
(-\operatorname{Re}(\lambda))^{\frac{1}{2}}\|C R(\lambda, A)\| \leq D
$$

for any complex number $\lambda$ with $\operatorname{Re}(\lambda)<0$. To check this, just observe that we have

$$
C R(\lambda, A) x=-\int_{0}^{\infty} e^{\lambda t} C T_{t} x d t
$$

by the Laplace formula (2.7). Hence if (4.5) holds, we have

$$
\begin{aligned}
\|C R(\lambda, A) x\| & \leq \int_{0}^{\infty} e^{\operatorname{Re}(\lambda) t}\left\|C T_{t} x\right\| d t \leq\left(\int_{0}^{\infty} e^{2 \operatorname{Re}(\lambda) t} d t\right)^{\frac{1}{2}}\left(\int_{0}^{\infty}\left\|C T_{t} x\right\|^{2} d t\right)^{\frac{1}{2}} \\
& \leq \frac{M}{\sqrt{2}(-\operatorname{Re}(\lambda))^{\frac{1}{2}}}\|x\| .
\end{aligned}
$$

To find conditions on $A$ that ensure that the converse implication '(4.6) $\Rightarrow(4.5)$ ' holds true is one of the most important questions in the area. The question whether this holds true for any $A$ remained open for a while under the name of 'Weiss conjecture'. This conjecture was disproved by Jacob, Partington and Pott [24] in the case when $H=K=\ell^{2}$, and by Jacob and Zwart [25] in the case when $H=\ell^{2}$ and $K=\mathbb{C}$.

Here we consider again the special case when $\left(T_{t}\right)_{t \geq 0}$ is a bounded analytic semigroup. In this case it is easy to check that $C=A^{\frac{1}{2}}$ satisfies (4.6). On the other hand, it follows from Lemma 4.1 (1) that $A^{\frac{1}{2}}$ is admissible for $A$ if and only if $A$ has a square function estimate (in the sense of Remark $3.3(2)$ ). These conditions turn out to characterize the validity of the implication '(4.6) $\Rightarrow(4.5)$ '.

TheOREM 4.4 ([34]). Let A be a sectorial operator of type $\omega<\frac{\pi}{2}$ on some Hilbert space $H$. The following assertions are equivalent.

(i) $A^{\frac{1}{2}}$ is admissible for $A$.

(ii) A has a square function estimate. 
(iii) A continuous linear operator $C: D(A) \rightarrow K$ is admissible for $A$ if and only if there is a constant $D>0$ such that $(-\operatorname{Re}(\lambda))^{\frac{1}{2}}\|C R(\lambda, A)\| \leq D$ for any complex number $\lambda$ with $\operatorname{Re}(\lambda)<0$.

Proof. The equivalence between (i) and (ii), and the implication '(iii) $\Rightarrow$ (i)', follow from the discussion before the theorem. We now prove '(ii) $\Rightarrow$ (iii)', which is the main implication. We assume that $A$ has a square function estimate and we let $C: D(A) \rightarrow K$ be an operator satisfying (4.6). This implies that for any $\gamma \in\left(\frac{\pi}{2}, \pi\right)$, there is a constant $D^{\prime}$ such that

$$
|\lambda|^{\frac{1}{2}}\|C R(\lambda, A)\| \leq D^{\prime}, \quad \lambda \in \mathbb{C} \backslash \Sigma_{\gamma} .
$$

Using Remark 2.6, we may assume that $A$ is 1 -1. In that case, $A^{-\frac{1}{2}}$ is a well-defined densely defined operator. Hence given any $x \in H$ and any $t>0$, we may write

$$
C e^{-t A} x=C A^{-\frac{1}{2}} A^{\frac{1}{2}} e^{-t A} x=t^{-\frac{1}{2}} C A^{-\frac{1}{2}} F_{0}(t A) x,
$$

where $F_{0}(z)=z^{\frac{1}{2}} e^{-z}$. Let $\varphi$ and $\psi$ be defined by

$$
\varphi(z)=\frac{z^{\frac{1}{4}}}{1+z} \quad \text { and } \quad \psi(z)=z^{\frac{1}{4}}(1+z) e^{-z} .
$$

Then $F_{0}=\varphi \psi, \psi \in H_{0}^{\infty}\left(\Sigma_{\omega+}\right)$, and $\varphi \in H_{0}^{\infty}\left(\Sigma_{\theta}\right)$ for any $\theta \in(0, \pi)$. By (4.8), we have

$$
\begin{aligned}
\int_{0}^{\infty}\left\|C T_{t} x\right\|^{2} d t & =\int_{0}^{\infty}\left\|C A^{-\frac{1}{2}} \varphi(t A) \psi(t A) x\right\|^{2} \frac{d t}{t} \\
& \leq \int_{0}^{\infty}\left\|C A^{-\frac{1}{2}} \varphi(t A)\right\|^{2}\|\psi(t A) x\|^{2} \frac{d t}{t} \\
& \leq \sup _{t>0}\left\{\left\|C A^{-\frac{1}{2}} \varphi(t A)\right\|^{2}\right\}\|x\|_{\psi}^{2} .
\end{aligned}
$$

To prove (4.5), it therefore suffices to show that

$$
\sup _{t>0}\left\{\left\|C A^{-\frac{1}{2}} \varphi(t A)\right\|\right\}<\infty .
$$

Let $\gamma>\frac{\pi}{2}$ be any angle. One can show that

$$
C A^{-\frac{1}{2}} \varphi(t A)=\frac{1}{2 \pi i} \int_{\Gamma_{\gamma}} z^{-\frac{1}{2}} \varphi(t z) C R(z, A) d z
$$

(see [34] for details). Applying (4.7), we deduce that for some constant $D^{\prime \prime}$ not depending on $t>0$, we have

$$
\left\|C A^{-\frac{1}{2}} \varphi(t A)\right\| \leq D^{\prime \prime} \int_{\Gamma_{\gamma}}|\varphi(t z)|\left|\frac{d z}{z}\right|=\int_{\Gamma_{\gamma}}|\varphi(z)| \frac{d z}{z} \mid .
$$

This is finite, by (2.4), which concludes the proof.

REMARK 4.5. In the paper [21], Bernhard Haak and the author introduced a variant of admissibility, called $\alpha$-admissibility. Let $\alpha>-1$ be a real number. An operator $C$ is $\alpha$-admissible if it satisfies an estimate of the form

$$
\int_{0}^{\infty} t^{\alpha}\|C T(t) x\|^{2} d t \leq M^{2}\|x\|^{2} .
$$


Let $\beta \in(-1,3)$ be such that $k=\frac{\alpha+\beta}{2}$ is a nonnegative integer. Let $A$ be a sectorial operator of type $<\frac{\pi}{2}$ that has a square function estimate. We show that an operator $C$ is $\alpha$-admissible if and only if there exists a constant $D>0$ such that

$$
\left\|(-\operatorname{Re}(\lambda))^{\frac{1+\beta}{2}} C R(\lambda, A)^{k+1}\right\| \leq D, \quad \lambda \in \mathbb{C}, \operatorname{Re}(\lambda)<0 .
$$

Moreover, the square function estimate is a necessary condition for this equivalence to hold.

\section{Square functions on $L^{p}$-spaces}

5.A. Main results on $L^{p}$-spaces. Let $1 \leq p<\infty$ be a number, let $\Omega$ be an arbitrary measure space, and consider the Banach space $X=L^{p}(\Omega)$. Given a sectorial operator $A$ of type $\omega \in(0, \pi)$ on $L^{p}(\Omega)$ and $F \in H_{0}^{\infty}\left(\Sigma_{\omega+}\right)$, we let

$$
\|x\|_{F}=\left\|\left(\int_{0}^{\infty}|F(t A) x|^{2} \frac{d t}{t}\right)^{\frac{1}{2}}\right\|_{L^{p}(\Omega)}, \quad x \in L^{p}(\Omega) .
$$

More precisely, let $x_{F}$ be the continuous function from $\mathbb{R}_{+}^{*}$ into $X=L^{p}(\Omega)$ defined by $x_{F}(t)=F(t A) x$. Let $H_{0}=L^{2}\left(\mathbb{R}_{+}^{*} ; \frac{d t}{t}\right)$. Then $\|x\|_{F}$ is defined as the norm of $x_{F}$ in the Hilbert space valued $L^{p}$-space $L^{p}\left(\Omega ; H_{0}\right)$, if $x_{F}$ belongs to that space, and $\|x\|_{F}=\infty$ otherwise. These square functions were introduced in [11] (see also Subsection 5.4 below). They obviously extend the ones on Hilbert space (see Section 4) that we recover when $p=2$. Our main objective in this subsection is to present analogs of Theorems 3.1 and 3.2 on $L^{p}$.

TheOREm 5.1 ([35]). Let $A$ be an $R$-sectorial operator of $R$-type $\omega \in(0, \pi)$ on a space $X=L^{p}(\Omega)$, with $1 \leq p<\infty$. Assume that $A$ has dense range. Let $F$ and $G$ be nonzero functions in $H_{0}^{\infty}\left(\Sigma_{\theta}\right)$, for some $\theta>\omega$.

(1) There is a constant $K>0$ such that for any $f \in H^{\infty}\left(\Sigma_{\theta}\right)$,

$$
\|f(A) x\|_{F} \leq K\|f\|_{\infty, \theta}\|x\|_{G}, \quad x \in L^{p}(\Omega) .
$$

(2) There is a constant $K>0$ such that

$$
K^{-1}\|x\|_{G} \leq\|x\|_{F} \leq K\|x\|_{G}, \quad x \in L^{p}(\Omega) .
$$

This generalization of Theorem 3.1 can be proved following the same approach as the one presented for the latter result in Section 3. See [35] for details. We do not know if this result remains true without assuming that $A$ is $R$-sectorial.

Assume that $A$ is a sectorial operator of type $\omega \in(0, \pi)$ on $X=L^{p}(\Omega)$, with $1 \leq p$ $<\infty$. Given any $F \in H_{0}^{\infty}\left(\Sigma_{\omega+}\right)$, we say that $A$ satisfies a square function estimate $\left(\mathcal{S}_{F}\right)$ if there is a constant $K>0$ such that

$$
\|x\|_{F} \leq K\|x\|, \quad x \in L^{p}(\Omega) .
$$

It clearly follows from the previous result that if $A$ is $R$-sectorial of $R$-type $\omega$, then $A$ satisfies $\left(\mathcal{S}_{F}\right)$ for some $F \in H_{0}^{\infty}\left(\Sigma_{\omega+}\right) \backslash\{0\}$ if and only if it satisfies $\left(\mathcal{S}_{F}\right)$ for all such $F$.

If $p \neq 1$, then $A^{*}$ is a sectorial operator of type $\omega \in(0, \pi)$ on $X^{*}=L^{p^{\prime}}(\Omega)$, where $p^{\prime}=p /(p-1)$ is the conjugate number of $p$. For any $G \in H_{0}^{\infty}\left(\Sigma_{\omega+}\right)$, we will say that $A$ satisfies $\left(\mathcal{S}_{G}^{*}\right)$ if $A^{*}$ satisfies a square function estimate with respect to $G$. 
THEOREM 5.2 ([11]). Let $A$ be a sectorial operator of type $\omega \in(0, \pi)$ on $X=L^{p}(\Omega)$, with $1 \leq p<\infty$. Let $\theta>\omega$.

(1) If A has a bounded $H^{\infty}\left(\Sigma_{\theta}\right)$ functional calculus, then $A$ satisfies a square function estimate $\left(\mathcal{S}_{F}\right)$ for any $F \in H_{0}^{\infty}\left(\Sigma_{\theta+}\right)$.

(2) Assume that $p \neq 1$. There exists a pair $(F, G)$ of nonzero functions in $H_{0}^{\infty}\left(\Sigma_{\omega+}\right)$ such that:

- If A satisfies $\left(\mathcal{S}_{F}\right)$ and $\left(\mathcal{S}_{G}^{*}\right)$, then $A$ has a bounded $H^{\infty}\left(\Sigma_{\theta}\right)$ functional calculus.

- If $A$ is $1-1$ and satisfies $\left(\mathcal{S}_{G}^{*}\right)$, then there is a constant $K>0$ such that

$$
\|x\| \leq K\|x\|_{F}, \quad x \in X .
$$

These fundamental results are due to Cowling, Doust, McIntosh, and Yagi [11]. They generalize the equivalence '(i) $\Leftrightarrow$ (ii)' and the implication '(i) $\Leftrightarrow$ (iii)' of Theorem 3.2. However their proofs are more difficult than the analogous ones on Hilbert space and require subtle decompositions of analytic functions in the spirit of harmonic analysis. These difficulties arise from the existence of bounded sets of operators on $L^{p}$ which are not $R$-bounded. A version of Theorem 3.2 on $L^{p}$-spaces goes as follows.

Corollary 5.3. Let $A$ be a 1-1 sectorial operator on $X=L^{p}(\Omega)$, with $1<p<\infty$, and let $\omega \in(0, \pi)$. The following assertions are equivalent.

(i) For any $\theta \in(\omega, \pi)$, A has a bounded $H^{\infty}\left(\Sigma_{\theta}\right)$ functional calculus.

(ii) For every $F, G$ in $H_{0}^{\infty}\left(\Sigma_{\omega+}\right)$, A satisfies $\left(\mathcal{S}_{F}\right)$ and $\left(\mathcal{S}_{G}^{*}\right)$.

(iii) The operator $A$ is $R$-sectorial of $R$-type $\omega$ and for every nonzero $F \in H_{0}^{\infty}\left(\Sigma_{\omega+}\right)$, there is a constant $K>0$ such that

$$
K^{-1}\|x\| \leq\|x\|_{F} \leq K\|x\|, \quad x \in X .
$$

Proof. Recall that $A^{*}$ has a bounded $H^{\infty}\left(\Sigma_{\theta}\right)$ functional calculus if and only if $A$ has one. Hence the equivalence between (i) and (ii) follows from Theorem 5.2. Since $X=L^{p}(\Omega)$ has property $(\Delta)$, the equivalence with (iii) follows from Theorems 2.7, 5.1, and 5.2.

EXAmPle 5.4. Square functions originate in harmonic analysis and it is worthwhile to mention a few classical ones, that are used e.g. in Stein's book [52]. For any $k \geq 1$, let

$$
G_{k}(z)=z^{k} e^{-z}, \quad z \in \mathbb{C} .
$$

Then $G_{k} \in H_{0}^{\infty}\left(\Sigma_{\omega+}\right)$ for any $\omega<\frac{\pi}{2}$. Hence if $A$ is a sectorial operator on $L^{p}(\Omega)$ of type $\omega<\frac{\pi}{2}$, then $G_{k}$ gives rise to a square function associated with $A$. Indeed if $\left(T_{t}\right)_{t \geq 0}$ is the bounded analytic semigroup generated by $-A$, we have

$$
G_{k}(t A) x=t^{k} A^{k} e^{-t A} x=(-t)^{k} \frac{\partial^{k}}{\partial t^{k}}\left(T_{t} x\right), \quad x \in L^{p}(\Omega) .
$$

Hence the corresponding square function is

$$
\|x\|_{G_{k}}=\left\|\left(\int_{0}^{\infty} t^{2 k-1}\left|\frac{\partial^{k}}{\partial t^{k}}\left(T_{t} x\right)\right|^{2} d t\right)^{1 / 2}\right\|_{L^{p}(\Omega)}, \quad x \in L^{p}(\Omega) .
$$


On the other hand, let us define

$$
\sigma_{t} x=\frac{1}{t} \int_{0}^{t} T_{s} x d s, \quad t>0, x \in L^{p}(\Omega) .
$$

Then consider the holomorphic function

$$
\Phi(z)=\frac{1-e^{-z}}{z}-e^{-z}, \quad z \in \mathbb{C} .
$$

It is easy to check that $\Phi \in H_{0}^{\infty}\left(\Sigma_{\omega+}\right)$ for any $\omega<\frac{\pi}{2}$. Furthermore, one has

$$
\Phi(t A) x=t \frac{\partial}{\partial t}\left(\sigma_{t} x\right), \quad t>0 .
$$

This is left as an exercice for the reader, details can be found e.g. in [26, Chapter 10]. Thus

$$
\|x\|_{\Phi}=\left\|\left(\int_{0}^{\infty} t\left|\frac{\partial}{\partial t}\left(\sigma_{t} x\right)\right|^{2} d t\right)^{1 / 2}\right\|_{L^{p}(\Omega)}
$$

is a square function associated to $A$. We will come back to these examples in the next subsection.

5.B. Diffusion semigroups. The following class of semigroups was considered by Stein in [52]. It provides a good illustration of the theory developed so far. A noncommutative analog will be presented in Section 6 below. Let $\Omega$ be a measure space and let $\left(T_{t}\right)_{t \geq 0}$ be a $c_{0}$-semigroup on $L^{2}(\Omega)$. We say that $\left(T_{t}\right)_{t \geq 0}$ is a diffusion semigroup on $\Omega$ if the following two conditions hold:

(I) For any $t \geq 0$, for any $1 \leq p \leq \infty$, and for any $x \in L^{p} \cap L^{2}, T_{t} x$ belongs to $L^{p}$, and $\left\|T_{t} x\right\|_{p} \leq\|x\|_{p}$.

(II) For any $t \geq 0, T_{t}: L^{2} \rightarrow L^{2}$ is a selfadjoint operator.

Let $t \geq 0$ be any nonnegative real number. By $(\mathrm{I}), T_{t}$ uniquely extends to a contractive operator $L^{p}(\Omega) \rightarrow L^{p}(\Omega)$ if $1 \leq p<\infty$, and to a contractive $w^{*}$-continuous operator $L^{\infty}(\Omega) \rightarrow L^{\infty}(\Omega)$. We will keep the notation $T_{t}$ for all these realizations. For any $1 \leq$ $p<\infty$, the resulting contraction semigroup $\left(T_{t}\right)_{t \geq 0}$ on $L^{p}(\Omega)$ is strongly continuous (see [54]). In the sequel, we let $-A_{p}$ denote the infinitesimal generator of the realization of $\left(T_{t}\right)_{t \geq 0}$ on $L^{p}(\Omega)$. It follows from [52, III.2] that $A_{p}$ is sectorial of type $\pi\left|\frac{1}{p}-\frac{1}{2}\right|$. The main result of this subsection is the following.

Theorem 5.5 (Cowling [10]). Let $\left(T_{t}\right)_{t \geq 0}$ be a diffusion semigroup on $\Omega$ and let $-A_{p}$ be the generator of $\left(T_{t}\right)_{t \geq 0}$ on $L^{p}(\Omega)$. Then for any $1<p<\infty$, and for any $\theta>\pi\left|\frac{1}{p}-\frac{1}{2}\right|$, the operator $A_{p}$ has a bounded $H^{\infty}\left(\Sigma_{\theta}\right)$ functional calculus.

We will sketch a proof of Cowling's Theorem, pointing out some of the general ideas. The first step is a transference result, whose general form is as follows.

Proposition 5.6 (Hieber-Prüss [22]). Let $X$ be a UMD Banach space, and let $\left(U_{t}\right)_{t \in \mathbb{R}}$ be a bounded $c_{0}$-group on $X$. Let $-B$ be the generator of this group. Then $B$ has a bounded $H^{\infty}\left(\Sigma_{\theta}\right)$ functional calculus for any $\theta>\frac{\pi}{2}$. 
Let $\left(V_{t}\right)_{t}$ be the translation group on $L^{2}(\mathbb{R} ; X)$, and let $-\mathcal{B}_{X}=\frac{d}{d t}$ denote its generator. The proof of Proposition 5.6 consists, using a transference method, in showing that for any $b \in L^{1}(\mathbb{R}+; d t)$, we have

$$
\left\|\int_{0}^{\infty} b(t) U_{t} d t\right\| \leq K^{2}\left\|\int_{0}^{\infty} b(t) V_{t} d t\right\|,
$$

where $K=\sup _{s}\left\|U_{s}\right\|$. Then using (2.8), this implies that $\|f(B)\| \leq K^{2}\left\|f\left(\mathcal{B}_{X}\right)\right\|$ for any $f \in H_{0}^{\infty}\left(\Sigma_{\frac{\pi}{2}+}\right)$. Therefore it suffices to show that $\mathcal{B}_{X}$ has a bounded $H^{\infty}\left(\Sigma_{\theta}\right)$ functional calculus for any $\theta>\frac{\pi}{2}$. Using the hypothesis that $X$ is UMD, this follows from McConnel's generalization of Mikhlin's multiplier theorem from [41].

Proof of Theorem 5.5. We only give a sketch, based on the following dilation property. Let $\left(T_{t}\right)_{t \geq 0}$ be a diffusion semigroup on $\Omega$, and let $1<p<\infty$. According to [14], there exist a measure space $\Omega^{\prime}$, an isometry $J: L^{p}(\Omega) \rightarrow L^{p}\left(\Omega^{\prime}\right)$, a contraction $Q: L^{p}\left(\Omega^{\prime}\right) \rightarrow L^{p}(\Omega)$, and a $c_{0}$-group $\left(U_{t}\right)_{t \in \mathbb{R}}$ of isometries on $L^{p}\left(\Omega^{\prime}\right)$, such that

$$
T_{t}=Q U_{t} J, \quad t \geq 0 .
$$

(This is continuous version of Ackoglu's famous dilation theorem for subpositive contractions on $L^{p}$.)

Let $-B$ be the generator of $\left(U_{t}\right)_{t}$. Using either (2.8) or (2.5), it follows from (5.4) that $\left\|f\left(A_{p}\right)\right\| \leq\|f(B)\|$ for any $f \in H_{0}^{\infty}\left(\Sigma_{\frac{\pi}{2}+}\right)$. Since $L^{p}\left(\Omega^{\prime}\right)$ is UMD, we deduce from Proposition 5.6 that $A_{p}$ has a bounded $H^{\infty}\left(\Sigma_{\theta}\right)$ functional calculus for any $\theta>\frac{\pi}{2}$.

By (II), the operator $A_{2}$ is a positive selfadjoint operator on $L^{2}(\Omega)$. Hence it admits a bounded $H^{\infty}\left(\Sigma_{\nu}\right)$ functional calculus for any $\nu>0$. Combining these two results, and using complex interpolation, one can deduce that $A_{p}$ actually has a bounded $H^{\infty}\left(\Sigma_{\theta}\right)$ functional calculus for any $\theta>\pi\left|\frac{1}{p}-\frac{1}{2}\right|$.

We refer the reader to [32], [12], [13], [9], and [57] for variants and extensions of Cowling's Theorem.

Combining Theorem 5.2 (1), Theorem 5.5, and the examples from 5.4, one finds extensions of various square function estimates established by Stein (see [52, p.115 and p.120]). Namely if $\left(T_{t}\right)_{t \geq 0}$ is a diffusion semigroup on $\Omega$, then for any $1<p<\infty$ there is a constant $K_{p}>0$ such that

$$
\left\|\left(\int_{0}^{\infty} t^{2 k-1}\left|\frac{\partial^{k}}{\partial t^{k}}\left(T_{t} x\right)\right|^{2} d t\right)^{1 / 2}\right\|_{p} \leq K_{p}\|x\|_{p}
$$

and

$$
\left\|\left(\int_{0}^{\infty} t\left|\frac{\partial}{\partial t}\left(\sigma_{t} x\right)\right|^{2} d t\right)^{1 / 2}\right\|_{p} \leq K_{p}\|x\|_{p}
$$

for any $x \in L^{p}(\Omega)$, where $\sigma_{t}$ is the averaging function defined by (5.2). In fact if $A_{p}$ is $1-1$, then the above inequalities can be reversed, by Corollary 5.3.

6. Extension to noncommutative $L^{p}$-spaces. In this section, we present analogs of the previous results for noncommutative $L^{p}$-spaces, as well as an illustration. All results from this section are taken from joint work with Marius Junge and Quanhua Xu 
$[26,27]$. Throughout this section, we let $\mathcal{M}$ denote a semifinite von Neumann algebra equipped with a normal semifinite faithful trace. For any $1 \leq p \leq \infty$, we let $L^{p}(\mathcal{M})$ denote the associated noncommutative $L^{p}$-space. We refer the reader e.g. to [50] for all necessary information on these spaces. We merely recall that if $\mathcal{M}=B\left(\ell^{2}\right)$ is the algebra of all bounded operators on $\ell^{2}$ equipped with its usual trace, then the corresponding noncommutative $L^{p}$-spaces are the Schatten spaces. On the other hand, if $\mathcal{M}$ is commutative and if we represent $\mathcal{M}=L^{\infty}(\Omega)$ for some measure space $\Omega$ in the usual way, then $L^{p}(\mathcal{M})=L^{p}(\Omega)$, the classical (commutative) $L^{p}$-space over $\Omega$. We also recall that if $1<p<\infty, L^{p}(\mathcal{M})$ is a UMD Banach space [6], and that $L^{p}(\mathcal{M})^{*}=L^{p^{\prime}}(\mathcal{M})$, where $p^{\prime}=p /(p-1)$ is the conjugate number of $p$.

6.A. Square functions and $H^{\infty}$ functional calculus on noncommutative $L^{p}$-spaces. We will define square functions for sectorial operators on $L^{p}(\mathcal{M})$. In order to extend (5.1), we need Hilbert space valued noncommutative $L^{p}$-spaces. Let $H$ be a Hilbert space and let $e \in H$ with $\|e\|=1$. For $1 \leq p<\infty$, we consider the embedding

$$
L^{p}(\mathcal{M}) \otimes H \hookrightarrow L^{p}(\mathcal{M} \bar{\otimes} B(H)),
$$

obtained by taking any $x \otimes a$ to $x \otimes(a \otimes e)$ for any $x \in L^{p}(\mathcal{M})$ and $a \in H$, and then extending by linearity. Then we let $L^{p}\left(\mathcal{M} ; H_{c}\right)$ be the completion of $L^{p}(\mathcal{M}) \otimes H$ for the norm induced by $L^{p}(\mathcal{M} \bar{\otimes} B(H))$. It turns out that this definition does not depend on $e$. The index ' $c$ ' is for 'column'. Indeed, it is easy to check that if $\left(e_{1}, \ldots, e_{n}\right)$ is an orthonormal family of $H$ and $a_{1}, \ldots, a_{n}$ belong to $L^{p}(\mathcal{M})$, then

$$
\left\|\sum_{k} a_{k} \otimes e_{k}\right\|_{L^{p}\left(M ; H_{c}\right)}=\left\|\left(\sum_{k} a_{k}^{*} a_{k}\right)^{\frac{1}{2}}\right\|_{L^{p}(M)}=\left\|\left[\begin{array}{cccc}
a_{1} & 0 & \cdots & 0 \\
\vdots & \vdots & & \vdots \\
a_{n} & 0 & \cdots & 0
\end{array}\right]\right\|_{L^{p}\left(M_{n}(M)\right)} .
$$

Likewise we may consider the embedding $L^{p}(\mathcal{M}) \otimes H \hookrightarrow L^{p}(\mathcal{M} \bar{\otimes} B(\bar{H}))$ obtained by taking $x \otimes a$ to $x \otimes(\bar{e} \otimes \bar{a})$ for any $x \in L^{p}(\mathcal{M})$ and $a \in H$, and we let $L^{p}\left(\mathcal{M} ; H_{r}\right)$ be the completion of $L^{p}(\mathcal{M}) \otimes H$ for the resulting norm. Again this does not depend on $e$. The index ' $\mathrm{r}$ ' is for 'row', and the analog of $(6.1)$ is

$$
\left\|\sum_{k} a_{k} \otimes e_{k}\right\|_{L^{p}\left(M ; H_{r}\right)}=\left\|\left(\sum_{k} a_{k} a_{k}^{*}\right)^{\frac{1}{2}}\right\|_{L^{p}(M)}=\left\|\left[\begin{array}{ccc}
a_{1} & \ldots & a_{n} \\
0 & \ldots & 0 \\
\vdots & & \vdots \\
0 & \cdots & 0
\end{array}\right]\right\|_{L^{p}\left(M_{n}(M)\right)} .
$$

We apply these definitions to the Hilbert space $H=H_{0}=L^{2}\left(\Omega_{0}\right)$, where $\Omega_{0}=$ $\left(\mathbb{R}_{+}^{*}, \frac{d t}{t}\right)$. Let $A$ be a sectorial operator of type $\omega \in(0, \pi)$ on $L^{p}(\mathcal{M})$, with $1 \leq p<\infty$. For any $F$ in $H_{0}^{\infty}\left(\Sigma_{\omega+}\right)$, we define square functions associated with $A$ by letting

$$
\|x\|_{F, c}=\|t \mapsto F(t A) x\|_{L^{p}\left(M ; L^{2}\left(\Omega_{0}\right)_{c}\right)} \quad \text { and } \quad\|x\|_{F, r}=\|t \mapsto F(t A) x\|_{L^{p}\left(M ; L^{2}\left(\Omega_{0}\right)_{r}\right)}
$$

for any $x \in L^{p}(\mathcal{M})$. More precisely, let $x_{F}(t)=F(t A) x$ for any $t>0$. Then $\|x\|_{F}$ is defined as the norm of $x_{F}$ in $L^{p}\left(\Omega ; H_{c}\right)$, if $x_{F}$ belongs to that space, and $\|x\|_{F, c}=\infty$ otherwise. The convention for $\|x\|_{F, r}$ is similar. 
Next we define symmetrized square functions by letting

$$
\|x\|_{F}=\max \left\{\|x\|_{F, c},\|x\|_{F, r}\right\} \quad \text { if } 2 \leq p<\infty ;
$$

and

$$
\begin{aligned}
& \|x\|_{F}=\inf \left\{\left\|u_{1}\right\|_{L^{p}\left(M ; L^{2}\left(\Omega_{0}\right)_{c}\right)}+\left\|u_{2}\right\|_{L^{p}\left(M ; L^{2}\left(\Omega_{0}\right)_{r}\right)}:\right. \\
& \left.u_{1}+u_{2}=F(\cdot A) x\right\} \quad \text { if } 1<p \leq 2,
\end{aligned}
$$

the infimum running over all possible decompositions of $x_{F}=F(\cdot A) x$ into a sum $u_{1}+u_{2}$ of measurable functions $u_{j}: \Omega_{0} \rightarrow L^{p}(\mathcal{M})$. It is not hard to see that if $\mathcal{M}$ is commutative, then the above definition of \|\|$_{F}$ coincides with (5.1). As with the noncommutative Khintchine inequalities due to Lust-Piquard (see $[40,50]$ ), the definitions depend upon whether $p \geq 2$ or $p<2$.

Although they are quite involved, these definitions are close to (5.1). Indeed one can show that if $p \geq 2$, then we have

$$
\|x\|_{F, c}=\sup _{0<\alpha<\beta<\infty}\left\|\int_{\alpha}^{\beta}(F(t A) x)^{*}(F(t A) x) \frac{d t}{t}\right\|_{L^{\frac{p}{2}(M)}}^{\frac{1}{2}}, \quad x \in L^{p}(\mathcal{M}) .
$$

Here $(F(t A) x)^{*}$ stands for the adjoint of $F(t A) x$ regarded as an operator on the Hilbert space on which $\mathcal{M}$ is acting. Likewise, we obtain $\|x\|_{F, r}$ by changing $(F(t A) x)^{*}(F(t A) x)$ into $(F(t A) x)(F(t A) x)^{*}$. These formulas should be regarded as 'continuous versions' of (6.1) and (6.2).

As in the commutative case, we will say that $A$ satisfies a square function estimate $\left(\mathcal{S}_{F}\right)$ if there is a constant $K>0$ such that $\|x\|_{F} \leq K\|x\|$ for any $x \in X=L^{p}(\mathcal{M})$.

TheOREM 6.1 ([26]). Theorems 5.1, 5.2, and Corollary 5.3 extend to the case when $X=$ $L^{p}(\mathcal{M})$ is a noncommutative $L^{p}$-space.

6.B. Noncommutative diffusion semigroups. Let $\left(T_{t}\right)_{t \geq 0}$ be a $c_{0}$-semigroup on the Hilbert space $L^{2}(\mathcal{M})$. Extending the terminology from the previous section, we say that $\left(T_{t}\right)_{t \geq 0}$ is a (noncommutative) diffusion semigroup on $\mathcal{M}$ if conditions (I) and (II) from 5.B hold true. As in the commutative case, they imply that $\left(T_{t}\right)_{t \geq 0}$ extends to a contractive $c_{0}$-semigroup on $L^{p}(\mathcal{M})$ for every $1<p<\infty$. Again we let $-A_{p}$ denote the generator of the realization of $\left(T_{t}\right)_{t \geq 0}$ on $L^{p}(\mathcal{M})$. We let

$$
\omega_{p}=\pi\left|\frac{1}{p}-\frac{1}{2}\right|
$$

Arguing as in [52, III.2], one has the following basic result.

LEMMA 6.2. Let $\left(T_{t}\right)_{t \geq 0}$ be a noncommutative diffusion semigroup on $L^{2}(\mathcal{M})$ and for any $1<p<\infty$, let $-A_{p}$ denote the generator of $\left(T_{t}\right)_{t \geq 0}$ on $L^{p}(\mathcal{M})$. Then $A_{p}$ is a sectorial operator of type $\omega_{p}$.

We do not know whether Theorem 5.5 extends to noncommutative $L^{p}$-spaces. The obstacle is the lack of an analog of the dilation property (5.4) in the noncommutative setting. Our main 'general' result for noncommutative diffusion semigroups is Theorem 6.3 below. Before stating it, we recall that the space $L^{p}(\mathcal{M})$ has a natural positive cone 
$L^{p}(\mathcal{M})_{+}$. Thus we say that a linear mapping $T: L^{p}(\mathcal{M}) \rightarrow L^{p}(\mathcal{M})$ is positive it it maps $L^{p}(\mathcal{M})_{+}$into itself. Then for an integer $n \geq 2$, we say that $T$ is $n$-positive if

$$
I_{M_{n}} \otimes T: L^{p}\left(M_{n}(\mathcal{M})\right) \rightarrow L^{p}\left(M_{n}(\mathcal{M})\right)
$$

is positive. Finally we say that $T$ is completely positive if it is $n$-positive for all $n$. See e.g. [46] for more on completely positive maps.

THEOREM $6.3([26])$. Let $\left(T_{t}\right)_{t \geq 0}$ be a noncommutative diffusion semigroup on $\mathcal{M}$ and for any $1<p<\infty$, let $-A_{p}$ denote the generator of $\left(T_{t}\right)_{t \geq 0}$ on $L^{p}(\mathcal{M})$. Assume moreover that $T_{t}$ is 2-positive for any $t \geq 0$. Then $A_{p}$ is $R$-sectorial of $R$-type $\omega_{p}$.

The paper [26] contain various examples of noncommutative diffusion semigroups satisfying the conclusions of Theorem 5.5. In the next subsection, we present one of them.

6.C. Noncommutative Fourier multipliers. Let $n \geq 1$ be an integer, and let $G=\mathbb{F}_{n}$ be the free group with $n$ generators denoted by $c_{1}, \ldots, c_{n}$. We let $e$ be the unit element of $G$, and we let $\left(\delta_{g}\right)_{g \in G}$ denote the canonical basis of the space $\ell_{G}^{2}$ of all square summable families of complex numbers indexed by $G$. Then we let $\lambda: G \rightarrow B\left(\ell_{G}^{2}\right)$ be the left regular representation of $G$, defined by $\lambda(g) \delta_{h}=\delta_{g h}$ for any $g, h \in G$. We recall that the group von Neumann algebra $V N(G) \subset B\left(\ell_{G}^{2}\right)$ is defined as the von Neumann algebra on $\ell_{G}^{2}$ generated by the $*$-algebra

$$
\mathcal{P}=\operatorname{Span}\{\lambda(g): g \in G\} .
$$

We let $\tau$ be the normalized trace on $V N(G)$ defined by $\tau(x)=\left\langle x\left(\delta_{e}\right), \delta_{e}\right\rangle$ for any $x \in$ $V N(G)$. We will consider $\mathcal{M}=V N(G)$ and the noncommutative $L^{p}$-spaces associated with the above trace. It is easy to check that for any $1 \leq p<\infty, \mathcal{P} \subset L^{p}(V N(G))$ is a dense subspace.

Since $G$ is a free group, any $g \in G$ has a unique decomposition of the form

$$
g=c_{i_{1}}^{k_{1}} c_{i_{2}}^{k_{2}} \cdots c_{i_{p}}^{k_{p}}
$$

where $p \geq 0$ is an integer, each $i_{j}$ belongs to $\{1, \ldots, n\}$, each $k_{j}$ is a nonzero integer, and $i_{j} \neq i_{j+1}$ if $1 \leq j \leq p-1$. The case when $p=0$ corresponds to the unit element $g=e$. By definition, the length of $g$ is defined as

$$
|g|=\left|k_{1}\right|+\cdots+\left|k_{p}\right| .
$$

This is the number of factors in the above decomposition of $g$.

For any $t \geq 0$, we let $T_{t}: \mathcal{P} \rightarrow \mathcal{P}$ be the linear mapping defined by letting

$$
T_{t}(\lambda(g))=e^{-t|g|} \lambda(g), \quad g \in G .
$$

These operators were introduced by Haagerup in [19], where he shows that each $T_{t}$ uniquely extends to a $w^{*}$-continuous unital completely positive map $T_{t}: V N(G) \rightarrow$ $V N(G)$. Moreover, $T_{t}$ also extends to a contractive, selfadjoint operator on $L^{2}(\mathcal{M})$. Then it is easy to check that $\left(T_{t}\right)_{t \geq 0}$ is a completely positive diffusion semigroup in the sense of the previous subsection. Note that if $n=1$, then $G=\mathbb{Z}$, and $\left(T_{t}\right)_{t \geq 0}$ is the classical Poisson semigroup on $L^{\infty}(\mathbb{T})$. For this reason, we call $\left(T_{t}\right)_{t \geq 0}$ the noncommutative Poisson 
semigroup on $\mathcal{M}=V N\left(\mathbb{F}_{n}\right)$. (We note in passing that there are other noncommutative versions of the Poisson transform in the literature, see e.g. [3].)

THEOREm 6.4 ([26]). Let $\left(T_{t}\right)_{t \geq 0}$ be the noncommutative Poisson semigroup on $V N\left(\mathbb{F}_{n}\right)$. For any $1<p<\infty$, let $-A_{p}$ be the generator of $\left(T_{t}\right)_{t \geq 0}$ on $L^{p}\left(V N\left(\mathbb{F}_{n}\right)\right)$. Then for any $\theta>\omega_{p}$, the operator $A_{p}$ has a bounded $H^{\infty}\left(\Sigma_{\theta}\right)$ functional calculus.

Proof. (Sketch.) The proof of this theorem owes a lot to Stein's original approach to (commutative) diffusion semigroups. Using noncommutative martingales (in the sense of [51]), we show that for any $1<p<\infty, A_{p}$ satisfies a square function estimate $\left(\mathcal{S}_{\Phi}\right)$, where $\Phi$ is the holomorphic function defined by (5.3). Since $A_{p}^{*}=A_{p^{\prime}}$, this shows that $A_{p}$ both satisfies $\left(\mathcal{S}_{\Phi}\right)$ and $\left(\mathcal{S}_{\Phi}^{*}\right)$. By Theorem 6.3 and by the noncommutative version of Theorem 5.1 provided by Theorem 6.1 , all square functions \|\|$_{F}$ for $A_{p}$ defined with a function $F \in H_{0}^{\infty}\left(\Sigma_{\omega_{p}+}\right) \backslash\{0\}$ are equivalent. Hence $A_{p}$ satisfies $\left(\mathcal{S}_{F}\right)$ and $\left(\mathcal{S}_{G}^{*}\right)$ for any $F, G$ in $H_{0}^{\infty}\left(\Sigma_{\omega_{p}+}\right)$. By the noncommutative version of Corollary 5.3 provided by Theorem 6.1, we deduce that $A_{p}$ has a bounded $H^{\infty}\left(\Sigma_{\theta}\right)$ functional calculus for any $\theta>\omega_{p}$.

It follows from the above theorem and its proof that (5.5) and (5.6) have analogs for the noncommutative Poisson semigroup. For example using (6.5) we obtain that if $p \geq 2$ and if $\left(T_{t}\right)_{t \geq 0}$ is the noncommutative Poisson semigroup on $V N\left(\mathbb{F}_{n}\right)$, then for any integer $k \geq 1$, there is a constant $K>0$ such that

$$
\left\|\int_{\alpha}^{\beta} t^{2 k-1}\left(\frac{\partial^{k}}{\partial t^{k}}\left(T_{t} x\right)\right)^{*}\left(\frac{\partial^{k}}{\partial t^{k}}\left(T_{t} x\right)\right) d t\right\|_{\frac{p}{2}}^{\frac{1}{2}} \leq K\|x\|_{p}
$$

for any $0<\alpha<\beta<\infty$ and any $x \in L^{p}\left(V N\left(\mathbb{F}_{n}\right)\right)$.

It clearly follows from (6.7) that $\mathcal{P} \subset D\left(A_{p}\right)$ and that $A_{p}(\lambda(g))=|g| \lambda(g)$ for any $g \in G$ and any $1<p<\infty$. Then

$$
\left[f\left(A_{p}\right)\right](\lambda(g))=f(|g|) \lambda(g), \quad g \in G,
$$

for every $f \in H_{0}^{\infty}\left(\Sigma_{\omega_{p}+}\right)$. Applying Theorem 6.4 we therefore deduce the following.

Corollary 6.5. Let $1<p<\infty$ and let $f \in H^{\infty}\left(\Sigma_{\theta}\right)$ for some $\theta>\omega_{p}$. Then there is a constant $K>0$ such that for every finitely supported family $\left(\alpha_{g}\right)_{g \in G \backslash\{e\}}$ of complex numbers, we have

$$
\left\|\sum_{g} \alpha_{g} f(|g|) \lambda(g)\right\|_{p} \leq K\left\|\sum_{g} \alpha_{g} \lambda(g)\right\|_{p} .
$$

In this statement, we may regard the family $\{f(|g|)\}_{g \in G \backslash\{e\}}$ as a 'noncommutative Fourier multiplier', and the corollary says that this multiplier is bounded on $L^{p}\left(V N\left(\mathbb{F}_{n}\right)\right)$ provided that $f \in H^{\infty}\left(\Sigma_{\theta}\right)$ for some $\theta>\omega_{p}$.

7. Further extensions. In Subsections 5.A and 6.A, we gave various results showing strong connections between square functions and $H^{\infty}$ functional calculus for sectorial operators on either commutative or noncommutative $L^{p}$-spaces. Some of these results turn out to extend quite naturally to sectorial operators defined on subspaces of certain Banach lattices. These results, taken from a joint work with Florence Lancien [31], will be explained shortly. Then we will present an abstract approach initiated and developed 
by Kalton and Weis in [30], which allows an extension of square functions and their connections with $H^{\infty}$ functional calculus to a large class of Banach spaces.

7.A. Banach lattices with a finite cotype and their subspaces. Let $\Lambda$ be a Banach lattice with a finite cotype (see e.g. [39]). This class includes classical (= commutative) $L^{p}$-spaces for any $1 \leq p<\infty$. Let $X \subset \Lambda$ be (closed) subspace, and let $A$ be a sectorial operator of type $\omega \in(0, \pi)$ on $X$. For any $F \in H_{0}^{\infty}\left(\Sigma_{\omega+}\right)$, we define

$$
\|x\|_{F}=\left\|\left(\int_{0}^{\infty}|F(t A) x|^{2} \frac{d t}{t}\right)^{\frac{1}{2}}\right\|_{\Lambda}, \quad x \in X .
$$

The meaning of this definition is similar to the one of (5.1). Indeed for any $x \in X$, we let $x_{F}(t)=F(t A) x$ for $t>0$. Then we let $H_{0}=L^{2}\left(\mathbb{R}_{+}^{*} ; \frac{d t}{t}\right)$, and $\|x\|_{F}$ is defined as the norm of $x_{F}$ in $\Lambda\left(H_{0}\right)$, if $x_{F}$ belongs to that space, and $\|x\|_{F}=\infty$ otherwise. The fact that $A$ is defined only on $X$ does not cause any trouble in this definition. However (when $X$ is reflexive) the adjoint operator $A^{*}$ does not necessarily act on a subspace of a Banach lattice. Thus (7.1) does not provide us with any square function associated with $A^{*}$. In consequence, we cannot expect any analog of Theorem 5.2 (2), say, in this context. This situation will be clarified in the next two subsections. For the moment we will content ourselves with the following.

TheOREm 7.1 ([31]). Let $A$ be a sectorial operator on $X \subset \Lambda$, where $\Lambda$ is a Banach lattice with a finite cotype. Assume that $A$ has dense range.

(1) Assume that $A$ is $R$-sectorial of $R$-type $\omega \in(0, \pi)$. Let $F$ and $G$ be nonzero functions in $H_{0}^{\infty}\left(\Sigma_{\theta}\right)$, for some $\theta>\omega$.

- There is a constant $K>0$ such that for any $f \in H_{0}^{\infty}\left(\Sigma_{\theta}\right)$,

$$
\|f(A) x\|_{F} \leq K\|f\|_{\infty, \theta}\|x\|_{G}, \quad x \in X .
$$

- There is a constant $K>0$ such that

$$
K^{-1}\|x\|_{G} \leq\|x\|_{F} \leq K\|x\|_{G}, \quad x \in X .
$$

(2) Assume that $A$ admits a bounded $H^{\infty}\left(\Sigma_{\theta}\right)$ functional calculus for some $\theta>\omega$. Then for any nonzero $F$ in $H_{0}^{\infty}\left(\Sigma_{\theta+}\right)$, there is a constant $K>0$ such that

$$
K^{-1}\|x\|_{F} \leq\|x\| \leq K\|x\|_{F}, \quad x \in X .
$$

Any subspace $X$ of a Banach lattice with finite cotype has property $(\Delta)$, hence Theorem 2.7 applies to it. It therefore follows from the above statement that the equivalence '(i) $\Leftrightarrow$ (iii)' in Corollary 5.3 holds true on $X$.

Let $N \geq 1$ be an integer, and let $R_{1}, \ldots, R_{N}$ denote the Riesz transforms on $\mathbb{R}^{N}$. The classical real-variables Hardy space $H^{1}\left(\mathbb{R}^{N}\right)$ may be defined as

$$
H^{1}\left(\mathbb{R}^{N}\right)=\left\{h \in L^{1}\left(\mathbb{R}^{N}\right): R_{j}(h) \in L^{1}\left(\mathbb{R}^{N}\right) \text { for any } j=1, \ldots, N\right\},
$$

and it is a Banach space for the norm

$$
\|h\|_{H^{1}}=\|h\|_{1}+\sum_{j=1}^{N}\left\|R_{j}(h)\right\|_{1}, \quad h \in H^{1}\left(\mathbb{R}^{N}\right) .
$$


(See e.g. [53].) The mapping $h \mapsto\left(h, R_{1}(h), \ldots, R_{N}(h)\right)$ from $H^{1}\left(\mathbb{R}^{N}\right)$ into $Z=$ $\ell_{N+1}^{1}\left(L^{1}\left(\mathbb{R}^{N}\right)\right)$ is an isometry. Since the latter space is an $L^{1}$-space, we may therefore regard

$$
H^{1}\left(\mathbb{R}^{N}\right) \hookrightarrow Z \simeq L^{1}(\Omega)
$$

as a subspace of an $L^{1}$-space.

Let $A$ be a sectorial operator of type $\omega \in(0, \pi)$ on $H^{1}\left(\mathbb{R}^{N}\right)$, and let $F \in H_{0}^{\infty}\left(\Sigma_{\omega+}\right)$. One can check (see [31] for details) that under the above identification, the square function \|\|$_{F}$ associated with $A$ is given by

$$
\|h\|_{F}=[h]_{F}+\sum_{j=1}^{N}[h]_{R_{j} F}
$$

where we have defined

$$
[h]_{T F}=\left\|\left(\int_{0}^{\infty}|T(F(t A) h)|^{2} \frac{d t}{t}\right)^{1 / 2}\right\|_{1}
$$

for any $T: H^{1}\left(\mathbb{R}^{N}\right) \rightarrow L^{1}\left(\mathbb{R}^{N}\right)$ and any $h \in H^{1}\left(\mathbb{R}^{N}\right)$.

Corollary 7.2. Let $A$ be a sectorial operator with dense range on $H^{1}\left(\mathbb{R}^{N}\right)$. If the square functions associated with $A$ are given by (7.2), then $A$ satisfies (1) and (2) in Theorem 7.1.

7.B. Square functions on general Banach spaces. In this short presentation, we mainly report on ideas and results from [30], to which we refer for proofs and developments. We give analogs of results from 5.A, 6.A, or 7.A on general Banach spaces. Precise connections with the latter sections will be given in the next Subsection 7.C. Throughout this section, we let $X$ be a Banach space with a finite cotype (see e.g. [39]). Let $\Omega_{0}=\left(\mathbb{R}_{+}^{*}, \frac{d t}{t}\right)$, and let $H_{0}=L^{2}\left(\Omega_{0}\right)$. We fix an orthonormal basis $\left(h_{j}\right)_{j \geq 1}$ on $H_{0}$. For any $\theta \in(0, \pi)$ and any $F \in H_{0}^{\infty}\left(\Sigma_{\theta}\right)$, we let

$$
F^{z}(t)=F(t z), \quad z \in \Sigma_{\theta}, t>0 .
$$

Each function $F^{z}$ belongs to $H_{0}$. Then for any integer $j \geq 1$, we set

$$
F_{j}(z)=\left\langle F^{z}, h_{j}\right\rangle_{H_{0}}=\int_{0}^{\infty} F(t z) \overline{h_{j}(t)} \frac{d t}{t}, \quad z \in \Sigma_{\theta} .
$$

Clearly each function $F_{j}$ belongs to $H^{\infty}\left(\Sigma_{\theta}\right)$.

Let $A$ be a sectorial operator of type $\omega \in(0, \pi)$ on $X$, and assume for simplicity that $A$ has dense range. For any $F \in H_{0}^{\infty}\left(\Sigma_{\omega+}\right)$, we define a square function by letting

$$
\|x\|_{F}=\sup _{n \geq 1}\left\|\sum_{j=1}^{n} \varepsilon_{j} F_{j}(A) x\right\|_{\operatorname{Rad}(X)}, \quad x \in X .
$$

Here we used the $\operatorname{Rad}(X)$-norms defined in 1.D.

Theorem 7.3. Let $X$ and $A$ be as above. Assume that $A$ is $R$-sectorial of $R$-type $\omega$. Let $F$ and $G$ be nonzero functions in $H_{0}^{\infty}\left(\Sigma_{\theta}\right)$, for some $\theta>\omega$.

(1) There is a constant $K>0$ such that for any $f \in H_{0}^{\infty}\left(\Sigma_{\theta}\right)$,

$$
\|f(A) x\|_{F} \leq K\|f\|_{\infty, \theta}\|x\|_{G}, \quad x \in X .
$$


(2) There is a constant $K>0$ such that

$$
K^{-1}\|x\|_{G} \leq\|x\|_{F} \leq K\|x\|_{G}, \quad x \in X .
$$

Let $F \in H_{0}^{\infty}\left(\Sigma_{\omega+}\right)$. As before, we say that $A$ satisfies a square function estimate $\left(\mathcal{S}_{F}\right)$ if there is a constant $K>0$ such that $\|x\|_{F} \leq K\|x\|$ for any $x \in X$. On the other hand, let $G \in H_{0}^{\infty}\left(\Sigma_{\omega+}\right)$; we say that $A$ satisfies a dual square function estimate $\left(\mathcal{S}_{G}^{d}\right)$ if there is a constant $K>0$ such that

$$
\left\|\sum_{j=1}^{n} F_{j}(A) x_{j}\right\|_{X} \leq K\left\|\sum_{j=1}^{n} \varepsilon_{j} x_{j}\right\|_{\operatorname{Rad}(X)}
$$

for any $n \geq 1$ and any $x_{1}, \ldots, x_{n}$ in $X$. Connections between these estimates and $H^{\infty}$ functional calculus are given by the following set of results.

Theorem 7.4. Let $X$ and $A$ be as above, and let $\theta>\omega$.

(1) If $A$ has a bounded $H^{\infty}\left(\Sigma_{\theta}\right)$ functional calculus, then $A$ satisfies a square function estimate $\left(\mathcal{S}_{F}\right)$, and a dual square function estimate $\left(\mathcal{S}_{G}^{d}\right)$ for any $F, G$ in $H_{0}^{\infty}\left(\Sigma_{\theta+}\right)$.

(2) There exists a pair $(F, G)$ of nonzero functions in $H_{0}^{\infty}\left(\Sigma_{\omega+}\right)$ such that:

- If A satisfies $\left(\mathcal{S}_{F}\right)$ and $\left(\mathcal{S}_{G}^{d}\right)$, then $A$ has a bounded $H^{\infty}\left(\Sigma_{\theta}\right)$ functional calculus.

- If A satisfies $\left(\mathcal{S}_{G}^{d}\right)$, then there is a constant $K>0$ such that

$$
\|x\| \leq K\|x\|_{F}, \quad x \in X .
$$

(3) If $A$ has a bounded $H^{\infty}\left(\Sigma_{\theta}\right)$ functional calculus, then there exist a function $F$ in $H_{0}^{\infty}\left(\Sigma_{\theta+}\right)$ and a constant $K>0$ such that

$$
K^{-1}\|x\| \leq\|x\|_{F} \leq K\|x\|, \quad x \in X .
$$

(4) The following assertions are equivalent.

(i) For any $\theta \in(\omega, \pi)$, A has a bounded $H^{\infty}\left(\Sigma_{\theta}\right)$ functional calculus.

(ii) For every $F, G$ in $H_{0}^{\infty}\left(\Sigma_{\omega+}\right)$, A satisfies $\left(\mathcal{S}_{F}\right)$ and $\left(\mathcal{S}_{G}^{d}\right)$.

REMARK 7.5. (1) In the above theorem, the main statements are (1) and (2). Indeed taking them for granted, (3) and (4) are straightforward consequences. If further $X$ has property $(\Delta)$, it follows from Theorems 2.7 and 7.3 that in (4) above, (i) and (ii) are also equivalent to:

(iii) The operator $A$ is $R$-sectorial of $R$-type $\omega$ and for every nonzero $F \in H_{0}^{\infty}\left(\Sigma_{\omega+}\right)$, there is a constant $K>0$ such that

$$
K^{-1}\|x\| \leq\|x\|_{F} \leq K\|x\|, \quad x \in X .
$$

(2) Assume that $X$ is reflexive and recall that $A^{*}$ is a sectorial operator of type $\omega$. Assume further that $X$ is $K$-convex (see e.g. [49]). Then there is an isomorphic identification $\operatorname{Rad}(X)^{*} \approx \operatorname{Rad}\left(X^{*}\right)$, and we easily deduce that for any $G \in H_{0}^{\infty}\left(\Sigma_{\omega+}\right), A$ has a dual square function estimate $\left(\mathcal{S}_{G}^{d}\right)$ if and only $A^{*}$ has a square function estimate $\left(\mathcal{S}_{\widetilde{G}}\right)$. Here $\widetilde{G}$ is defined as in (2.6). 
7.C. Comparing the abstract and the concrete approach. We let $X$ and $A$ be as in the previous subsection.

Assume that $X=H$ is a Hilbert space. Let $F \in H_{0}^{\infty}\left(\Sigma_{\omega+}\right)$. Since $\left(h_{j}\right)_{j}$ is an orthonormal basis of $H_{0}=L^{2}\left(\Omega_{0}\right)$, the mapping $U: L^{2}\left(\Omega_{0}\right) \rightarrow \ell^{2}$ taking any $h \in H$ to the sequence $\left(\left\langle h, h_{j}\right\rangle\right)_{j}$ is a unitary. Then $U \otimes I_{H}$ extends to another unitary

$$
U_{H}: L^{2}\left(\Omega_{0} ; H\right) \rightarrow \ell^{2}(H) .
$$

Let $x \in H$, and assume that $x_{F}(t)=F(t A) x$ belongs to $L^{2}\left(\Omega_{0} ; H\right)$. Then it is not hard to see that $U_{H}\left(x_{F}\right)=\left(F_{j}(A) x\right)_{j}$. Using $(2.10)$, we deduce that

$$
\begin{aligned}
\sup _{n \geq 1}\left\|\sum_{j=1}^{n} \varepsilon_{j} F_{j}(A) x\right\|_{\operatorname{Rad}(H)} & =\left(\sum_{j=1}^{\infty}\left\|F_{j}(A) x\right\|_{H}^{2}\right)^{\frac{1}{2}} \\
& =\left\|x_{F}\right\|_{L^{2}\left(\Omega_{0} ; H\right)}=\left(\int_{0}^{\infty}\|F(t A) x\|^{2} \frac{d t}{t}\right)^{\frac{1}{2}} .
\end{aligned}
$$

This shows that the square function defined by (7.3) coincides with the original one given by (3.1).

Assume now that $\Lambda$ is a Banach lattice with a finite cotype, and let $X \subset \Lambda$ be a subspace. In that case there exists a constant $K>0$ such that

$$
K^{-1}\left\|\sum_{k=1}^{n} \varepsilon_{k} x_{k}\right\|_{\operatorname{Rad}(X)} \leq\left\|\left(\sum_{k=1}^{n}\left|x_{k}\right|^{2}\right)^{1 / 2}\right\|_{\Lambda} \leq K\left\|\sum_{k=1}^{n} \varepsilon_{k} x_{k}\right\|_{\operatorname{Rad}(X)}
$$

for any finite family $x_{1}, \ldots, x_{n}$ in $X$ (see e.g. [39, 1.d.6]). Arguing as above and using (7.4) instead of (2.10), one can show that for any $F \in H_{0}^{\infty}\left(\Sigma_{\omega+}\right)$, the square function defined by (7.1) and the one defined by (7.3) are equivalent. This result includes the case when $X=L^{p}(\Omega)$ for $1 \leq p<\infty$.

Likewise, using the noncommutative Khintchine inequalities [40, 50], one can show that if $X=L^{p}(\mathcal{M})$ is a noncommutative $L^{p}$-space for some $1 \leq p<\infty$, then the square function defined by (7.3) and the one defined by (6.3) or (6.4) are equivalent for any $F \in H_{0}^{\infty}\left(\Sigma_{\omega+}\right)$.

Using these equivalence results, and Remark 7.5 (2), we can thus regard Theorems $3.1,3.2,5.1,5.2,6.1,7.1$, or Corollary 7.2, as consequences of Theorems 7.3 and 7.4.

Acknowledgements. The author wishes to express his gratitude to the referee for various suggestions which improved the presentation of the paper.

\section{References}

[1] D. Albrecht, X. T. Duong, and A. McIntosh, Operator theory and harmonic analysis, Proc. of CMA Canberra 34 (1996), 77-136.

[2] W. Arendt and $\mathrm{S}$. Bu, The operator valued Marcinkiewicz multiplier theorem and maximal regularity, Math. Zeit. 240 (2002), 311-343.

[3] A. Arias and G. Popescu, Noncommutative interpolation and Poisson transform, Israel J. Math. 115 (2000), 205-234. 
[4] P. Auscher, A. McIntosh, and A. Nahmod, Holomorphic functional calculi of operators, quadratic estimates and interpolation, Indiana Univ. Math. J. 46 (1997), 375-403.

[5] E. Berkson and T. A. Gillespie, Spectral decompositions and harmonic analysis on UMD Banach spaces, Studia Math. 112 (1994), 13-49.

[6] E. Berkson, T. A. Gillespie, and P. S. Muhly, Abstract spectral decompositions guaranteed by the Hilbert transform, Proc. London Math. Soc. 53 (1986), 489-517.

[7] D. Burkholder, Martingales and singular integrals in Banach spaces, in: Handbook of the Geometry of Banach Spaces, Vol. I, W. B. Johnson and J. Lindenstrauss (eds.), Elsevier, 2001, 233-269.

[8] P. Clément, B. De Pagter, F. Sukochev, and H. Witvliet, Schauder decompositions and multiplier theorems, Studia Math. 138 (2000), 135-163.

[9] P. Clément, and J. Prüss, Completely positive measures and Feller semigroups, Math. Annalen 287 (1990), 73-105.

[10] M. Cowling, Harmonic analysis on semigroups, Annals of Math. 117 (1983), 267-283.

[11] M. Cowling, I. Doust, A. McIntosh, and A. Yagi, Banach space operators with a bounded $H^{\infty}$ functional calculus, J. Austr. Math. Soc. (Series A) 60 (1996), 51-89.

[12] G. Dore and A. Venni, On the closedness of the sum of two closed operators, Math. Zeit. 196 (1987), 189-201.

[13] X. T. Duong, $H^{\infty}$ functional calculus of second order elliptic partial differential operators on $L^{p}$-spaces, Proc. of CMA Canberra 24 (1989), 91-102.

[14] G. Fendler, Dilations of one parameter semigroups of positive contractions on $L^{p}$-spaces, Canad. J. Math. 49 (1997), 736-748.

[15] E. Franks, Modified Cauchy kernels and functional calculus for operators on Banach space, J. Australian Math. Soc. Series A 63 (1997), 736-748.

[16] D. J. Garling, On martingales with values in a complex Banach space, Math. Proc. Cambridge Phil. Soc. 104 (1988), 399-406.

[17] J. A. Goldstein, Semigroups of Linear Operators and Applications, Oxford University Press, New York, 1985.

[18] P. Grabowsky and F. M. Callier, Admissible observation operators. Semigroup criteria of admissibility, Integral Equations and Operator Theory 25 (1996), 182-198.

[19] U. Haagerup, An example of a non nuclear $C^{*}$-algebra, which has the metric approximation property, Inventiones Math. 50 (1979), 279-293.

[20] U. Haagerup and G. Pisier, Factorization of analytic functions with values in noncommutative $L_{1}$-spaces and applications, Canad. J. Math. 41 (1989), 882-906.

[21] B. Haak and C. Le Merdy, $\alpha$-admissibility of observation and control operators, Houston J. Math. 31 (2005), 1153-1167.

[22] M. Hieber and J. Prüss, Functional calculi for linear operators in vector valued $L^{p}$-spaces via the transference principle, Adv. Diff. Equations 3 (1998), 847-872.

[23] B. Jacob and J. R. Partington, Admissibility of control and observation operators for semigroups: a survey, in: Current Trends in Operator Theory and its Applications, Oper. Theory Adv. Appl. 149, Birkhäuser, Basel, 2004, 199-221.

[24] B. Jacob, J. R. Partington, and S. Pott, Admissible and weakly admissible observation operators for the right shift semigroup, Proc. Edinburgh Math. Soc. 45 (2002), 353-362.

[25] B. Jacob and H. Zwart, Counterexamples concerning observation operators for $C_{0}-$ semigroups, SIAM J. Control Optimization 43 (2004), 137-153.

[26] M. Junge, C. Le Merdy, and Q. Xu, $H^{\infty}$ functional calculus and square functions on noncommutative $L^{p}$-spaces, Astérisque 306 (2006). 
[27] M. Junge, C. Le Merdy, and Q. Xu, Calcul fonctionnel et fonctions carrées dans les espaces $L^{p}$ non commutatifs, C. R. Acad. Sci. Paris Ser. I 337 (2003), 93-98.

[28] N. Kalton and G. Lancien, A solution to the problem of $L^{p}$-maximal regularity, Math. Zeit. 235 (2000), 559-568.

[29] N. Kalton and L. Weis, The $H^{\infty}$ calculus and sums of closed operators, Math. Annalen 321 (2001), 319-345.

[30] N. Kalton and L. Weis, $H^{\infty}$ functional calculus and square function estimates, preprint, 2005.

[31] F. Lancien and C. Le Merdy, Square functions and $H^{\infty}$ calculus on subspaces of $L^{p}$ and on Hardy spaces, Math. Z. 251 (2005), 101-115.

[32] D. Lamberton, Equations d'évolutions linéaires associées à des semigroupes de contractions dans les espaces $L^{p}$, J. Funct. Anal. 72 (1987), 252-262.

[33] C. Le Merdy, $H^{\infty}$ functional calculus and applications to maximal regularity, Pub. Math. Besançon 16 (1999), 41-77.

[34] C. Le Merdy, The Weiss conjecture for bounded analytic semigroups, J. London Math. Soc. 67 (2003), 715-738.

[35] C. Le Merdy, On square functions associated to sectorial operators, Bull. Soc. Math. France 132 (2004), 137-156.

[36] C. Le Merdy, On dilation theory for $c_{0}$-semigroups on Hilbert space, Indiana Univ. Math. J. 45 (1996), 945-959.

[37] C. Le Merdy, The similarity problem for bounded analytic semigroups on Hilbert space, Semigroup Forum 56 (1998), 205-224.

[38] C. Le Merdy, Similarities of $\omega$-accretive operators, Proc. of CMA Canberra 41 (2003), 84-95.

[39] J. Lindenstrauss and L. Tzafriri, Classical Banach Spaces II, Springer Verlag, Berlin, 1979.

[40] F. Lust-Piquard, Inégalités de Khintchine dans $C_{p}$, C. R. Acad. Sci. Paris Ser. I 303 (1986), 289-292.

[41] T. McConnel, On Fourier multiplier transformations of Banach valued functions, Trans. Amer. Math. Soc. 285 (1984), 739-757.

[42] A. McIntosh, Operators which have an $H^{\infty}$ functional calculus, Proc. of CMA Canberra 14 (1986), 210-231.

[43] A. McIntosh and A. Yagi, Operators of type $\omega$ without a bounded $H^{\infty}$ functional calculus, Proc. of CMA Canberra 24 (1989), 159-172.

[44] B. Sz-Nagy and C. Foiaş, Harmonic Analysis of Operators on Hilbert Space, Akademiai Kiadó, Budapest, 1970.

[45] V. I. Paulsen, Every completely polynomially bounded operator is similar to a contraction, J. Funct. Anal. 55 (1984), 1-17.

[46] V. I. Paulsen, Completely Bounded Maps and Operator Algebras, Cambridge Studies in Advanced Math. 78, Cambridge University Press, Cambridge, 2002.

[47] G. Pisier, A polynomially bounded operator on Hilbert space which is not similar to a contraction, J. Amer. Math. Soc. 10 (1997), 351-369.

[48] G. Pisier, Similarity Problems and Completely Bounded Maps, 2nd, expanded edition, Lecture Notes in Math. 1618, Springer Verlag, Berlin, 2001.

[49] G. Pisier, Probabilistic methods in the geometry of Banach spaces, in: Probability and Analysis, Lecture Notes in Math. 1206 (1986), 167-241. 
[50] G. Pisier and Q. Xu, Non-commutative $L^{p}$-spaces, in: Handbook of the Geometry of Banach Spaces, Vol. II, W. B. Johnson and J. Lindenstrauss (eds.), Elsevier, 2003, 14591517.

[51] G. Pisier and Q. Xu, Non-commutative martingale inequalities, Comm. Math. Phys. 189 (1997), 667-698.

[52] E. M. Stein, Topics in Harmonic Analysis Related to the Littlewood-Paley Theory, Ann. Math. Studies, Princeton University Press, 1985.

[53] E. M. Stein, Harmonic Analysis, Princeton University Press, 1993.

[54] J. Voigt, One-parameter semigroups acting simultaneously on different $L_{p}$-spaces, Bull. Soc. Roy. Sci. Liège 61 (1992), 465-470.

[55] G. Weiss, Two conjectures on the admissibility of control operators, in: Estimation and Control of Distributed Parameter Systems, Birkhäuser Verlag, 1991, 367-378.

[56] L. Weis, Operator valued Fourier multiplier theorems and maximal regularity, Math. Annalen 319 (2001), 735-758.

[57] L. Weis, A new approach to maximal $L_{p}$-regularity, in: Proc. of the 6th International Conference on Evolution Equations 1998, G. Lumer and L. Weis (eds.), Marcel Dekker, 2000, 195-214. 